\title{
A numerical investigation on impulse-induced nonlinear longitudinal waves in pantographic beams
}

\section{Emilio Turco}

Department of Architecture, Design and Urban planning (DADU), University of Sassari and International Research Center on Mathematics and Mechanics of Complex Systems (M\&MOCS), University of L'Aquila, Italy

\section{Emilio Barchiesi}

Department of Architecture, Design and Urban planning (DADU), University of Sassari, International Research Center on Mathematics and Mechanics of Complex Systems (M\&MOCS), University of L'Aquila, Italy and École Nationale d'Ingénieurs de Brest, ENIB, UMR CNRS 6027, IRDL, Brest, France

\section{Francesco dell'Isola}

Department of Civil, Construction-Architectural and Environmental Engineering (DICEAA) and International Research Center on Mathematics and Mechanics of Complex Systems (M\&MOCS), University of L'Aquila, Italy

Received 2 February 2021; accepted 29 March 2021

\begin{abstract}
This contribution presents the results of a campaign of numerical simulations aimed at better understanding the propagation of longitudinal waves in pantographic beams within the large-deformation regime. Initially, we recall the key features of a Lagrangian discrete spring model, which was introduced in previous works and that was tested extensively as capable of accurately forecasting the mechanical response of structures based on the pantographic motif, both in statics and dynamics. Successively, a stepwise integration scheme used to solve equations of motions is briefly discussed. The key content of the present contribution concerns the thorough presentation of some selected numerical simulations, which focus in particular on the propagation of stretch profiles induced by impulsive loads. The study takes into account different tests, by varying the number of unit cells, i.e., the total length of the system, spring stiffnesses, the shape of the impulse, as well as its properties such as duration and peak amplitude, and boundary conditions. Some conjectures about the form of traveling waves are formulated, to be confirmed by both further numerical simulations and analytical investigations.
\end{abstract}

Corresponding author:

Emilio Turco, Department of Architecture, Design and Urban planning (DADU), University of Sassari, via Garibaldi 35, Asilo Sella, Alghero (SS) 0704I, Italy.

Email: emilio.turco@uniss.it 


\section{Keywords}

Mechanical metamaterials, discrete models, nonlinear longitudinal waves, stepwise analysis, impulsive loads, pantographic structures

\section{Introduction}

Pantographic materials are a concrete example of how matter can be designed to exhibit some desired characteristics, mechanical characteristics in such a case. Although they were conceived for purposes related to theoretical mechanics, i.e., to provide motivations for the study of gradient elasticity theories [1] with extremely relevant non-local effects, pantographic materials show many characteristics that make them potentially useful for several applications, see, e.g., the recent review papers [2-6].

Owing also to this fact, in the last five years, intensive research was carried out which was devoted to: (i) formulating generalized continuum models able to synthetically forecast the mechanical response of pantographic structures both in statics and dynamics, see, e.g., [7-12]; (ii) designing experiments on fabrics manufactured by means of modern additive techniques such as 3D printing, see e.g. [13-20]; (iii) conceiving refined particle models able to reproduce experiments and to cast the base for constructing continuum models [21-26]. The largest part by far of research on pantographic materials has been focused on the analysis of small and large deformation static regimes. On the other hand, contributions dealing with the dynamical response of pantographic materials are rare in the scientific literature, some examples being [27-29]. The development of reliable modeling and computational tools addressing the dynamics of pantographic materials could accelerate the exploitation of such materials in cases of practical interest, where the influence of inertia forces cannot be neglected. Referring to pantographic beams, one could envisage studies analogous to those recently carried out in [30] and [31] about two-dimensional and three-dimensional Euler-Bernoulli beams, respectively. Pantographic beams, thanks to their highly compliant (actually zero-energy when perfect hinges are considered at nodes; see [7, 32-35]) accordion-like mechanism entailing relevant total elongation/compression, that can be triggered acting on a single cell, could be profitably employed to design underactuated exible robotic arms $[36,37]$.

The present study attempts to fill the aforementioned gap, namely the lack of a wide literature dealing with the dynamics of pantographic materials, building on top of the expertise developed and documented by a series of previous works related to the modeling of pantographic lattices by means of an intrinsically discrete model, largely inspired by Hencky's $\mathrm{PhD}$ thesis [38]. Pantographic lattices are modeled as assemblies of two (usually, but not necessarily, orthogonal) families of elastica beams exhibiting geometrical nonlinearities, which are mechanically approximated as chains of extensional springs with rotational springs in-between adjacent elements, see [39, 40]. Aimed at matching real experiments involving large displacements and deformations, a stepwise strategy based on Newton's method as the predictor and Riks's arc length as the corrector was implemented in statics.

Preliminarily to this contribution, a scarcely known stepwise time integration scheme, that was renamed Casciaro's scheme after its proponent, was revisited and adapted to the analysis of the intrinsically discrete modeling of pantographic beams mentioned previously. A useful starting point for readers interested in stepwise integration schemes, as opposed to time finite-element-based integrators [41], which can be easily adapted to deal with material nonlinearities and dissipative forces, is Wriggers's book [42, Chap. 6] which, in addition to providing an overview of the topic, also contains several references. Although in such a book there is no trace of Casciaro's stepwise integration scheme, this method displays some interesting peculiarities and, notwithstanding that it was proposed in the 1970s, it exploits modern ideas: (i) a quadratic B-spline interpolation in time for the displacements; (ii) impulse-momentum relationship in discrete form at each end of time intervals; (iii) an optimal choice of two parameters to produce the best, in a sense which will be clarified later, response in analyzing an elastic one-degree-of-freedom linear oscillator once the a priori chosen time step length is given. Roughly speaking, Casciaro's scheme ensures numerical stability for any given time step length, in addition to filtering the round-off errors and avoiding the beat phenomenon for large time step lengths. Clearly, when problems with nonlinearities are considered, there is no certainty about the fact that the dynamical response computed by Casciaro's integration scheme is still the best. However, this is valid for any integration scheme available in the literature.

In the present contribution, making use of the aforementioned tools, namely a simple multi-degree-offreedom discrete mechanical system and a stepwise integration scheme, we investigate the propagation in time 


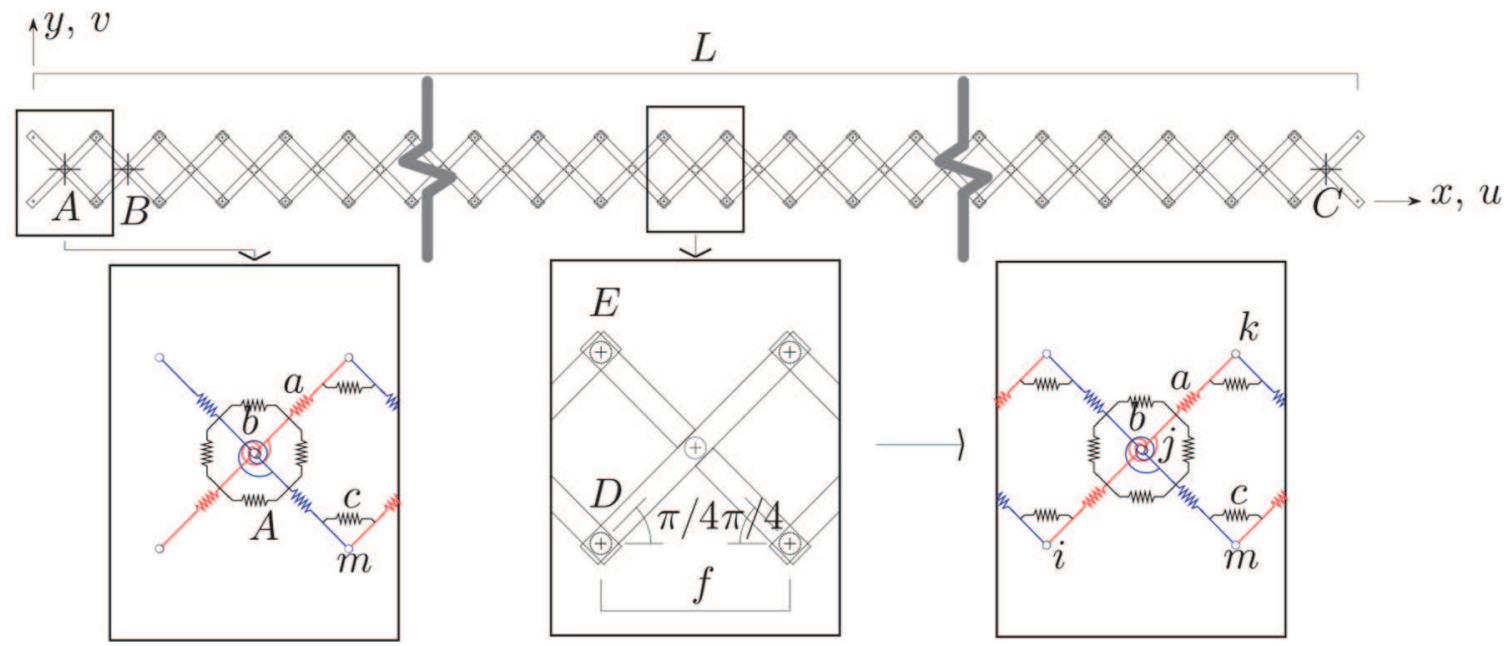

Figure I. Geometry, mechanical modeling, and constraints of the pantographic beam.

of longitudinal stretch waves induced by impulsive loads in pantographic beams, attempting to explore the most interesting features. Following this Introduction, we recall in Section 2 the intrinsically discrete spring mechanical model used for the description of pantographic beams in plane; successively, in Section 3, the stepwise solution strategy employed to solve the initial value problem is presented and discussed; in Section 4, the results of several numerical simulations are reported and thoroughly discussed before presenting some concluding remarks and outlining possible future challenges in Section 5.

\section{Mechanical modeling of pantographic beams}

Throughout this contribution, the system sketched in Figure 1, i.e., a pantographic beam, sometimes abbreviated as p-beam, is considered. The p-beam is formed by cross-like unit cells aligned on the horizontal $x$-axis, each one made up of two beam-like elements. Beam-like elements are connected through pivots, i.e., hinges with rotational springs. Past experience acquired in dealing with pantographic lattices formed by two families of orthogonal beams families of orthogonal beams [40, 43-45], non-orthogonal beams [46], and with pantographic beams [47, 48], sometimes modeled through more refined semi-discrete modeling based on classical beam theories [49-51], proves that the discrete spring system sketched in Figure 1 can describe in a simple and efficient, yet effective, way real prototypes realize by, e.g., additive manufacturing.

The key idea is to describe the mechanics of the p-beam by means of two kinds of mechanical interactions, the first being given by extensional springs and the second by rotational springs. These interactions aim at representing, in a very simple form, the resistance of beam-like beams to extension and bending, respectively. In addition, rotational springs are also employed to confer a torsional stiffness to the pivots connecting different beam-like elements. This straightforward mechanical description could obviously be improved in different ways. As an instance, the beam-like elements in each cross-like unit cell could be modeled as discrete Timoshenko beams, see [52], thus obtaining a more refined description. Furthermore, a $h$-refinement strategy such as that described in [53] could be used, where each beam-like element is described by several springs, both extensional and rotational, with the goal of improving the discrete approximation of beam-like elements as regarded as continuous Euler-Bernoulli beams. Clearly, the same concept could be applied should beam-like elements be regarded as generalizations of continuous Timoshenko beams. Referring to the simplest case, ${ }^{1}$ namely considering the beam-like elements as modeled by Euler-Bernoulli theory and avoiding the use of any $h$-refinement strategy, the strain energy stored in each spring can be classified among one of the following

$$
\begin{aligned}
\text { beam stretching energy: } & E_{a}=\frac{1}{2} a \Delta \ell^{2}, \\
\text { beam bending energy: } & E_{b}=b(1+\cos \beta), \\
\text { pivot torsional energy: } & E_{c}=\frac{1}{2} c\left(\gamma-\frac{\pi}{2}\right)^{2} .
\end{aligned}
$$


These springs are distinguished in Figure 1 by means of different colours: red and blue indicate extensional and bending (rotational) springs, whereas black indicates torsional (rotational) springs. Strain measures $\Delta \ell$, stretching, $\cos \beta$, bending, and $\gamma$, torsion, use only current positions of nodes within the p-beam or, equivalently, nodal displacements. ${ }^{2}$ More specifically, the strain measures are defined by

$$
\begin{array}{cl}
\text { stretching measure: } & \Delta \ell=\left\|p_{j}-p_{i}\right\|-\left\|P_{j}-P_{i}\right\|, \\
\text { bending measure: } & \cos \beta=\frac{\left\|p_{j}-p_{i}\right\|^{2}+\left\|p_{k}-p_{j}\right\|^{2}-\left\|p_{k}-p_{i}\right\|^{2}}{2\left\|p_{j}-p_{i}\right\|\left\|p_{k}-p_{j}\right\|}, \\
\text { shear strain measure: } & \gamma=\arccos \frac{\left\|p_{j}-p_{m}\right\|^{2}+\left\|p_{k}-p_{j}\right\|^{2}-\left\|p_{k}-p_{m}\right\|^{2}}{2\left\|p_{j}-p_{m}\right\|\left\|p_{k}-p_{j}\right\|},
\end{array}
$$

where $P_{i}$ and $p_{i}$ are the reference and current positions of the $i$ th node, respectively (an analogous convention holds for nodes labeled by indexes $j, k$, and $m$ ). We remark that, because for the generic $i$ th node we have $p_{i}=P_{i}+\mathbf{u}_{i}$, with $\mathbf{u}_{i}=\left(u_{i}, v_{i}\right)$ being its displacement, the stretching elementary contributions to the total strain energy depend only on the displacements $\mathbf{u}_{i}$ and $\mathbf{u}_{j}$, those due to bending depend only on $\mathbf{u}_{i}, \mathbf{u}_{j}$, and $\mathbf{u}_{k}$, whereas those for the torsion strain depend only on $\mathbf{u}_{j}, \mathbf{u}_{m}$, and $\mathbf{u}_{k}$. We also note that, as evident from (2), stiffnesses used for specifying the mechanics of the pantographic beam are denoted $a, b$, and $c$, for extensional, bending, and torsional springs, respectively. ${ }^{3}$

Note that the elementary strain energy contribution accumulated within each spring, concurring to the total strain energy accumulated within the p-beam as a whole system, which defines its mechanics, could take forms different from those utilized in (2). As an instance, different elementary bending energy contributions $E_{b}$ can be considered such as those reported in $[54,55]$. Indeed, the classical expression $2 E_{b}=\bar{b} \beta^{2}$ could equivalently be used, with the stiffness coefficient $\bar{b}$ clearly different, generally speaking, from $b$ in (2). By using an elementary Taylor's series expansion, it is straightforward to show that when $\beta$ lies in a small neighborhood of $\pi$, i.e., the value assumed by $\beta$ in the reference configuration, the two choices for $E_{b}$ are equivalent.

In conclusion, the total strain energy $E$ of the whole p-beam can be easily obtained by adding for all the elastic elements the elementary contributions listed in (1). We choose as Lagrangian configuration variables of the system the nodal displacements and collect them within the vector $\mathbf{u}$. We then define the structural reaction $\mathbf{s}$, in short the reaction, as the gradient of the total strain energy with respect to the Lagrangian variables

$$
\mathbf{s}(\mathbf{u})=\frac{\partial E}{\partial \mathbf{u}} .
$$

Furthermore, we define the so-called stiffness matrix $\mathbf{K}(\mathbf{u})$ as the Hessian of the total strain energy or, equivalently, the gradient of the reaction

$$
\mathbf{K}(\mathbf{u})=\frac{\partial \mathbf{s}}{\partial \mathbf{u}} .
$$

The gradient and the Hessian of the total strain energy of the p-beam are the only tools which are necessary to build a stepwise strategy to solve the nonlinear equilibrium equations ${ }^{4}$ in statics. Within this contribution we are dealing with the dynamical response of pantographic beams and, therefore, we shall introduce the total kinetic energy of the system. To this end, having in mind physical realizations of pantographic structures already studied in the past, we take into account two kind of contributions. The first relates with the beams, i.e., mass distributed along the beams, and the second one with the pivots, i.e., mass concentrated at pivots. More specifically, for a one-dimensional link having length $\ell_{e}$, the first contribution reads as

$$
\frac{1}{2} \int_{0}^{\ell_{e}} \rho_{e} A_{e} \mathbf{v}(x) \cdot \mathbf{v}(x) \mathrm{d} x=\frac{1}{2} \dot{\mathbf{u}}_{e} \cdot\left(\int_{0}^{\ell_{e}} \rho_{e} A_{e} \mathbf{B}_{e}^{T} \mathbf{B}_{e} \mathrm{~d} x\right) \dot{\mathbf{u}}_{e}=\frac{1}{2} \dot{\mathbf{u}}_{e} \cdot \mathbf{M}_{e} \dot{\mathbf{u}}_{e},
$$

where $\rho_{e}$ is its mass density, $A_{e}$ is its cross-section area, and $\mathbf{v}$ is the velocity vector. This latter vector can be written by using the matrix of the shape functions $\mathbf{B}_{e}$, which is linear in this case, and the Lagrangian velocities collected in the vector $\dot{\mathbf{u}}_{e}$. The matrix $\mathbf{B}_{e}$, which collects the shape functions, can be expressed using the local coordinate $0 \leq x \leq \ell_{e}$ as

$$
\mathbf{B}_{e}=\left[\begin{array}{cccc}
1-x / \ell_{e} & 0 & x / \ell_{e} & 0 \\
0 & 1-x / \ell_{e} & 0 & x / \ell_{e}
\end{array}\right]
$$


For links with uniform cross-section, some straightforward calculations give the expression of the elementary mass matrix contribution

$$
\mathbf{M}_{e}=\frac{1}{6} \rho_{e} A_{e} \ell_{e}\left[\begin{array}{llll}
2 & 0 & 1 & 0 \\
0 & 2 & 0 & 1 \\
1 & 0 & 2 & 0 \\
0 & 1 & 0 & 2
\end{array}\right],
$$

whereas the elementary mass matrix contribution due to the $p$ th pivot can be written as

$$
\mathbf{M}_{p}=\rho_{p} \frac{\pi d_{p}^{2}}{4} h\left[\begin{array}{ll}
1 & 0 \\
0 & 1
\end{array}\right],
$$

where $d_{p}$ and $h$ are the diameter and the height of the $p$ th cylindrical pivot, respectively. ${ }^{5}$ The elementary mass matrix contributions, both those related to the links (7) and those related to the pivots (8), are the only quantities that are necessary to assemble the global mass matrix $\mathbf{M}$ by a straightforward procedure which resembles, even if it generally less tedious, the standard one operated for assembling the (global) stiffness matrix, which has not been reported here.

Total strain and kinetic energies, as defined in the foregoing, allow to compute the mass matrix $\mathbf{M}$, which in the considered case can be recognized as constant, i.e., not depending on the current configuration, and the reaction $\mathbf{s}$. These are the only quantities which are strictly necessary to write down the final expression of the system of ordinary differential equations of motion (Euler-Lagrange equations, see [56]) governing the dynamics of the pantographic beam. In matrix form, the Euler-Lagrange equations of the considered system read as

$$
\mathbf{M u}(t)+\mathbf{s}(\mathbf{u}(t))-\mathbf{f}(t)=\mathbf{0},
$$

where, in addition to the Lagrangian parameters used for describing the motion, i.e., the displacement vector $\mathbf{u}$, the velocity vector $\dot{\mathbf{u}}$, and the acceleration vector $\ddot{\mathbf{u}}$, the external force vector $\mathbf{f}$ appears too.

\section{Solution of the nonlinear equations of motion by a stepwise strategy}

The technical literature is highly populated by contributions devoted to solving systems of nonlinear equations of motion. It is therefore a very difficult task to choose a small set of significant relatively recent contributions. In the present authors' opinion, some of them are given in [57-59]. Wriggers's book [42] is updated up to the first years of 2000 and provides a very good introduction to stepwise integration schemes. Starting from a (possibly non-uniform) time discretization that is deemed suitable for the underlying problem, a stepwise integration scheme aims at recovering the equilibrium path, i.e., the pair made up of time, $t$, and Lagrangian variables collected in the displacement $\mathbf{u}$ and velocity $\dot{\mathbf{u}}$ vectors, by solving a sequence of initial value problems. Roughly speaking, for each time interval $\Delta t$, one of the intervals which are employed to discretize the time domain until the time horizon, the solution at its right extreme point is computed starting from the solution at its left extreme. Clearly, choosing an appropriate length for time steps discretizing the time domain is crucial, especially for nonlinear problems, and, generally speaking, it requires both some preliminary analyses and a noteworthy experience on the analyst's side.

Numerical integration schemes that do not require, at least in principle, particularly heavy preliminary analyses should be preferable. Among those which are available, the numerical integration scheme presented by Casciaro [60] in 1975 appears attractive for its adaptability to the (a priori) choice of the time step length. However, even if it was published in an important journal such as Meccanica, Casciaro's scheme is still largely unknown to the scholars engaged in this research field. ${ }^{6}$

The main idea of Casciaro consists of the use of two free parameters which are being optimized to compute the best dynamical response for the a priori chosen time step length. More specifically, Casciaro observes that the numerical solution of the time evolution of a system is always affected by errors depending on the chosen time step length $\Delta t$. These errors may be triggered by choosing a time step length greater than a fraction of the fundamental (minimum) natural period of the considered system, thus leading to numerical instabilities. In addition, round-off errors introduced when the time step length is too small may prejudicate, even in a significant way, the correctness of the numerical solution. Finally, a too large time step length might introduce spurious beatings, that is beating produced by numerical errors, even for unconditionally stable schemes. 
Bearing in mind the aforementioned observations, Casciaro's algorithm is based on the following features: (i) within a time interval, displacements are interpolated in time by means of a quadratic interpolation law, where the interpolation data are given by the values of the displacement and velocity at the beginning and at the end of the time interval ${ }^{7}$; (ii) the momentum-impulse relationship in discrete form. The vector collecting nodal displacements at time $t$, belonging to the time interval $t_{1}-t_{0}$, can be written as

$$
\mathbf{u}(\xi)=\mathbf{u}_{0}+\beta_{0} \dot{\mathbf{u}}_{0}\left(2 \xi-\xi^{2}\right)+\beta_{1} \dot{\mathbf{u}}_{1} \xi^{2}, \quad \xi=\frac{t-t_{0}}{t_{1}-t_{0}},
$$

with $\mathbf{u}$ and $\dot{\mathbf{u}}$ being the displacement and velocity vectors, respectively. Subscripts 0 and 1 indicate the initial and the final time of the time interval, respectively. The weighting factors $\beta_{0}$ and $\beta_{1}$, which are such that $\beta_{0}+\beta_{1}=1$, are free and may be tuned optimally to improve the performances of the integration scheme. Evaluating the interpolation law 10 at the end of the time interval $(\xi=1)$, we compute the vector $\mathbf{u}_{1}$ as

$$
\mathbf{u}_{1}=\mathbf{u}_{0}+\beta_{0} \dot{\mathbf{u}}_{0}+\beta_{1} \dot{\mathbf{u}}_{1}
$$

The momentum-impulse relationship in discrete form is ${ }^{8}$

$$
\mathbf{M}\left(\dot{\mathbf{u}}_{1}-\dot{\mathbf{u}}_{0}\right)+\alpha_{0}\left(\mathbf{s}_{0}-\mathbf{f}_{0}\right)+\alpha_{1}\left(\mathbf{s}_{1}-\mathbf{f}_{1}\right)=\mathbf{0},
$$

where the momentum change $\mathbf{M}\left(\dot{\mathbf{u}}_{1}-\dot{\mathbf{u}}_{0}\right)$ and the average net impulse $\alpha_{0}\left(\mathbf{s}_{0}-\mathbf{f}_{0}\right)+\alpha_{1}\left(\mathbf{s}_{1}-\mathbf{f}_{1}\right)$ can be recognized. This contains the weighting factors $\alpha_{0}$ and $\alpha_{1}$, which are such that $\alpha_{0}+\alpha_{1}=1$, and that, once again, are free parameters that may be tuned to improve the performances of the integration scheme. Owing to the constraint relationships $\alpha_{0}+\alpha_{1}=1$ and $\beta_{0}+\beta_{1}=1$, only two free parameters, say $\alpha$ and $\beta$, among $\alpha_{0}, \alpha_{1}, \beta_{0}$, and $\beta_{1}$ are independent. These free parameters can be tuned optimally on the basis of $T_{1}$ (first natural period) and $T_{n}$ (last natural period) of the considered problem. In this regard, Casciaro [60] provides analytical expressions of the parameters $\alpha$ and $\beta$. More precisely, in the case $\Delta t<T_{n} / 2$ one has

$$
\begin{array}{ll}
\beta=-\alpha=\sqrt{-\frac{1}{4}+\frac{1}{\tau^{2}}-\frac{1+\sqrt{1+\tan ^{2} \tau}}{2 \tan ^{2} \tau}}, & 0<\tau<\frac{\pi}{2}, \\
\beta=-\alpha=\sqrt{-\frac{1}{4}+\frac{1}{\tau^{2}}-\frac{1-\sqrt{1+\tan ^{2} \tau}}{2 \tan ^{2} \tau}}, & \frac{\pi}{2}<\tau<\pi,
\end{array}
$$

being $\tau=2 \pi \Delta t / T_{n}$. Note that (13), when $\Delta t \rightarrow 0$, tends to $\beta=-\alpha=1 / \sqrt{6}$. Instead, when $\Delta t \rightarrow T_{n} / 2$, we obtain $\beta=-\alpha=1 / \pi$. Conversely, when $\Delta t>T_{n} / 2$, the optimal values are

$$
\begin{aligned}
& \alpha=-\frac{T_{n}}{2 \pi \Delta t}+\frac{c^{3}}{1+2 c^{3}}, \\
& \beta=\frac{T_{n}}{2 \pi \Delta t}+\frac{c^{3}}{1+2 c^{3}},
\end{aligned}
$$

being

$$
c=\frac{2 \Delta t-T_{n}}{T_{1}-T_{n}}
$$

Formulas (14) contain two additive terms: a hyperbola branch which matches the optimal values of $\alpha$ and $\beta$ at the end of the interval $\Delta t<T_{n} / 2$ and the ratio between two cubic polynomials, which tends, when $\Delta t \rightarrow \infty$, to the value $1 / 2$. Therefore, the corresponding values of $\alpha$ and $\beta$ are capable of reproducing a quasi-static solutions.

In [60], Casciaro does not furnish many details about the properties of the proposed integration scheme and does not report thoroughly computations leading him to its final formulation. This is probably the main reason at the basis of its lack of success. Actually, a more detailed description of the integration scheme can be found in [61], although both [60] and [61] contain some typos in the formulas. Recently, a more thorough analysis of the Casciaro's scheme has been carried out in [28], which presents some numerical examples and 
analytical computations providing insights into the method, specifically into the rationale leading to the optimal expressions (13) and (14).

At this point, after having introduced the geometry and mechanics of the pantographic beam as well as the equations of motion to be solved, we have described almost all the numerical tools which are necessary for the analysis of the dynamic problems considered within this contribution. What is missing is only a solution strategy able to get around the geometrical nonlinearities of the p-beam response, i.e., to solve for each discrete time nonlinear equilibrium equations. First, we expand the reaction $\mathbf{s}_{1}$ using a truncated Taylor series

$$
\mathbf{s}_{1}=\mathbf{s}\left(\mathbf{u}_{1}, \dot{\mathbf{u}}_{1}\right) \approx \mathbf{s}_{0}+\mathbf{K}_{0}\left(\mathbf{u}_{1}-\mathbf{u}_{0}\right)
$$

where $\mathbf{K}_{0}$ denotes the stiffness matrix computed at the beginning of the considered time interval. According to (4), we have

$$
\mathbf{K}_{0}=\left.\frac{\partial \mathbf{s}}{\partial \mathbf{u}}\right|_{\mathbf{u}_{0}}
$$

From (11) and (12), the displacement variables, i.e., $\mathbf{u}_{1}$, can be eliminated by making use of (11). Equations (11)-(12), along with the definition of the stiffness matrix (17) and the expansion (16), provide the necessary ingredients to apply a Newton-like algorithm to the considered problem. In short, starting from what we define as the solution reminder calculated at the $j$ th iteration

$$
\mathbf{r}_{j}=\mathbf{M}\left(\dot{\mathbf{u}}_{1, j}-\dot{\mathbf{u}}_{0}\right)+\alpha_{0}\left(\mathbf{s}_{0}-\mathbf{f}_{0}\right)+\alpha_{1}\left(\mathbf{s}_{1, j}-\mathbf{f}_{1}\right),
$$

a new estimate of the solution is found in a Newton-like way by the following recursive relation

$$
\dot{\mathbf{u}}_{1, j+1}=\dot{\mathbf{u}}_{1, j}-\mathbf{H}_{j}^{-1} \mathbf{r}_{j},
$$

where the iteration matrix $\mathbf{H}$ is defined as

$$
\mathbf{H}_{j}=\left.\nabla \mathbf{r}\right|_{j}=\mathbf{M}+\alpha_{1} \beta_{1} \mathbf{K}_{j} .
$$

The quantity $K_{j}$ in (20) is the matrix in (4) evaluated at the $j$ th step of the Newton-like iterations performed within the current time step. At this point, we have also presented all the tools which are necessary to solve the nonlinear equilibrium problem for each discrete time. We end this section with some remarks. The first concerns the use of a Newton-like scheme. It is well known that this method is not able to overcome limit points, i.e., configurations that turn the iteration matrix zero determinant. This is a formidable task in static problems, where the iteration matrix is the stiffness matrix. In such a case, the Newton algorithm plays the role of a predictor, which has to be complemented with that of a corrector such as, e.g., the arc-length method proposed by Riks [62]. Conversely, in the dynamic case, the iteration matrix includes also the mass matrix contribution, see (20), which regularizes the problem, making the use of a corrector not strictly required.

The second remark concerns the possible inclusion of dissipative phenomena. In such a case, what has been dealt with above can be easily adapted by considering dissipative forces, as an instance described as $\mathbf{d}=\mathbf{d}(\dot{\mathbf{u}}){ }^{9}$ It is enough to rewrite the definition of $\mathbf{r}_{j}$ including these forces as

$$
\mathbf{r}_{j}=\mathbf{M}\left(\dot{\mathbf{u}}_{1, j}-\dot{\mathbf{u}}_{0}\right)+\alpha_{0}\left(\mathbf{s}_{0}+\mathbf{d}_{0}-\mathbf{f}_{0}\right)+\alpha_{1}\left(\mathbf{s}_{1, j}+\mathbf{d}_{1}-\mathbf{f}_{1}\right)
$$

where a new, adapted, definition of the iteration matrix $\mathbf{H}_{j}$ must be used. It must indeed include, in addition to the mass and stiffness matrices, also the dissipation matrix $\mathbf{C}_{j}$. In formulas, the dissipation matrix is defined as

$$
\mathbf{C}_{j}=\left.\frac{\partial \mathbf{C}}{\partial \dot{\mathbf{u}}}\right|_{\dot{\mathbf{u}}_{j}},
$$

whereas the iteration matrix becomes

$$
\mathbf{H}_{j}=\left.\nabla \mathbf{r}\right|_{j}=\mathbf{M}+\alpha_{1} \beta_{1} \mathbf{K}_{j}+\alpha_{1} \mathbf{C}_{j} .
$$


Table I. Datasets of material parameters used in the present study.

\begin{tabular}{llllll}
\hline & & $f$ & $a$ & $b$ & $c$ \\
Dataset & Cells & $(\mathrm{mm})$ & $(\mathrm{N} / \mathrm{mm})$ & $(\mathrm{Nmm})$ & $(\mathrm{Nmm})$ \\
\hline 1 & 200 & 13 & 65,000 & 20,000 & 22,000 \\
2 & 1000 & 13 & 65,000 & 20,000 & 22,000 \\
\hline
\end{tabular}

\section{Numerical results}

\section{I. Preliminary analysis}

Before addressing the more challenging large-deformation regime, the results of a modal analysis are to be reported. These results allow to get an insight into the behavior of the considered system in the smalldeformation regime, around the reference configuration. This kind of analysis turns out to be especially useful to suitably tune the parameters controlling some features of the time-integration scheme, such as the weighting factors and the time step. A pantographic beam constituted by 200 unit cells was considered. Material parameters are reported in Table 1.

Kinematic conditions $u_{A}=v_{A}=v_{B}=0(u$ denotes longitudinal displacements and $v$ denotes transverse displacements) were enforced at nodes $A$ and $B$. Having calculated the mass matrix $\mathbf{M}$ and the stiffness matrix $\mathbf{K}$ in the initial configuration, natural frequencies (or, equivalently, periods) and modes were obtained by solving an eigenvalue problem. They are reported in Figure 2.

It is worth remarking that (see Figure 2) the only uniaxial (extensional) mode is the 10th (with period $T_{10}=0.0530 \mathrm{~s}$ ), where uniform extension of the pantographic beam is achieved through its characteristic accordion-like mechanism. All the remaining modes are flexural. Based on these data, the time step was chosen as $\Delta t=10^{-4} \mathrm{~s}$ whenever 200 cells, each having length $f=13 \mathrm{~mm}$, the stiffness values, $a, b$, and $c$, in Table 1, and clamped (on the left) boundary conditions were considered for the p-beam. Although most of the simulations reported in the sequel fulfill all these conditions, a few variations were explored. Clearly, any variation required a dedicated preliminary modal analysis, as natural periods and modes change when, generally speaking, the mass and stiffness matrices change. In the sequel, should the present analysis not hold, this will be signaled, and the most relevant results of the dedicated modal analysis will be reported.

Making use of formulas presented in the previous section, the first and last periods of the pantographic beam, $T_{1}=19.7 \mathrm{~s}$ and $T_{n}=3.3 \times 10^{-5} \mathrm{~s}$ in the present case (see Figure 2), respectively, were employed to select the optimal values of the two free parameters in the time integration scheme.

\subsection{Hammer test}

In this test, longitudinal vibrations of the pantographic beam (with no pre-stressed elements) as induced by a compressive impulsive longitudinal load applied to the central point of the right end cell, i.e., node $C$, see Figure 1 , are studied in the time domain. At first, results obtained employing as impulse the triangle-shaped function in Figure 3 are to be reported. The quantity $\bar{p}$ in Figure 3 is fixed to be $\bar{p}=-4000 \mathrm{~N}$. The influence of the impulse duration, denoted with $2 r$ in Figure 3, has been studied through a parametric analysis.

Each row of plots in Figure 4 reports, on the left, the evolution in time of the longitudinal displacement $u_{C}$, namely the displacement of node $C$. In the center, each row of plots in Figure 4 reports the evolution in time of the velocity $\dot{u}_{C}$. Finally, each row of plots in Figure 4 reports, on the right, the phase portrait of the displacement $u_{C}$, i.e., velocity $\dot{u}_{C}$ versus $u_{C}$ along the system's trajectory. Results reported in the first row of Figure 4 have been obtained for $2 r=0.01 \mathrm{~s}$, whereas results for the second and third rows of Figure 4 have been obtained for $2 r=0.005 \mathrm{~s}$ and $2 r=0.002 \mathrm{~s}$, respectively. It is worth remarking that the displacement $u_{C}$ in Figure 4(a) shows a periodic oscillating behavior around its initial value, namely zero.

Each plot in Figure 5 reports stroboscopically the longitudinal stretch profile $s_{w}$, namely the difference between longitudinal displacements of successive central nodes. Stroboscopic profiles correspond to $t=0.005 \mathrm{~s}$ (cyan), $0.01 \mathrm{~s}$ (red), $0.15 \mathrm{~s}$ (yellow), and $0.2 \mathrm{~s}$ (violet). The stretch is amplified by a factor of 100 to make the plots more readable. Each plot in Figure 5 corresponds to a different impulse duration $(2 r=0.01 \mathrm{~s}, 2 r=$ $0.005 \mathrm{~s}$, and $2 r=0.002 \mathrm{~s}$ ).

Initially, during the impulse, the pantographic beam undergoes, through the accordion-like mechanism, a seemingly uniform compression, $u_{C}$ becoming increasingly negative. As soon as the externally applied force 


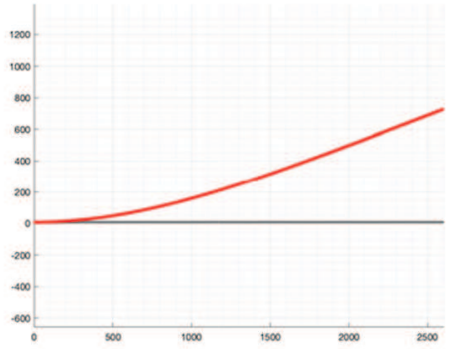

(a)

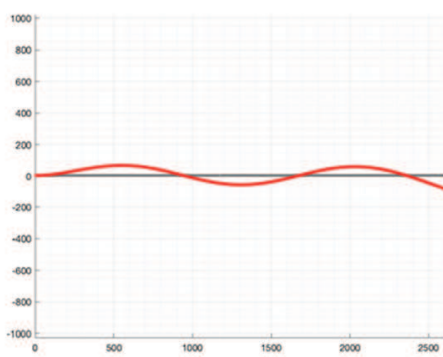

(d)

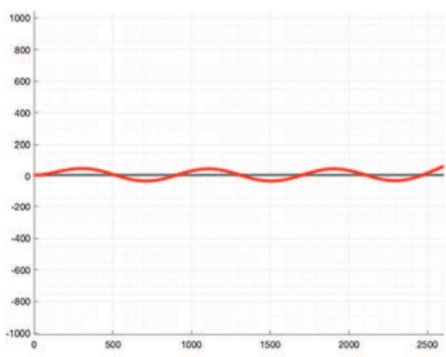

(g)

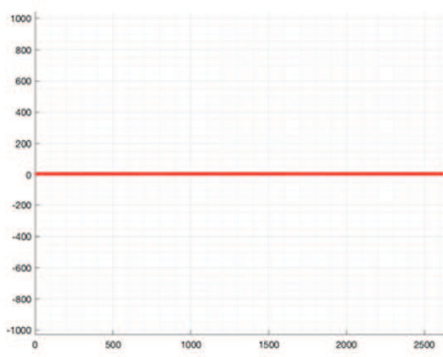

(j)

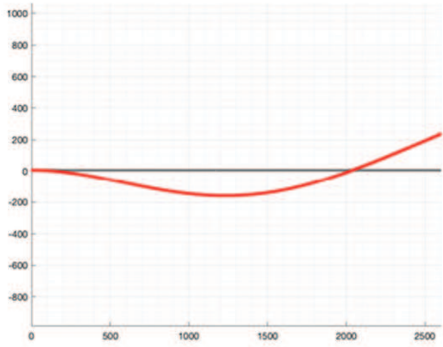

(b)

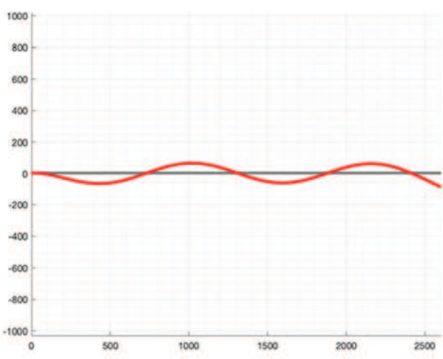

(e)

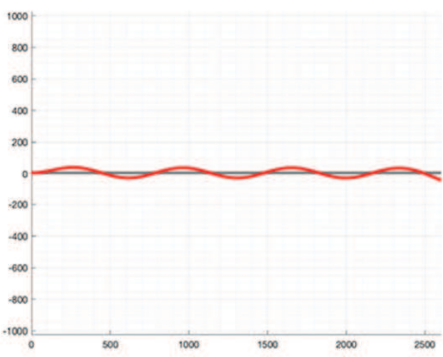

(h)

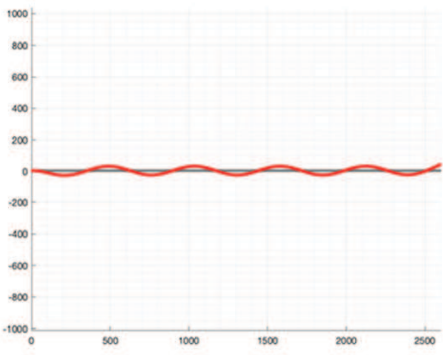

(k)

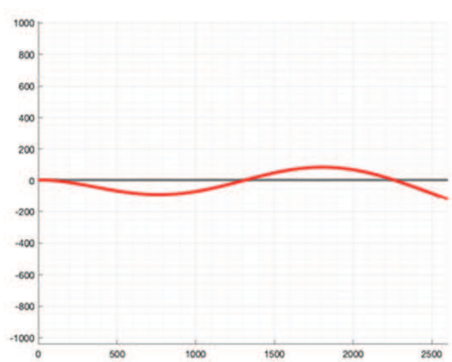

(c)

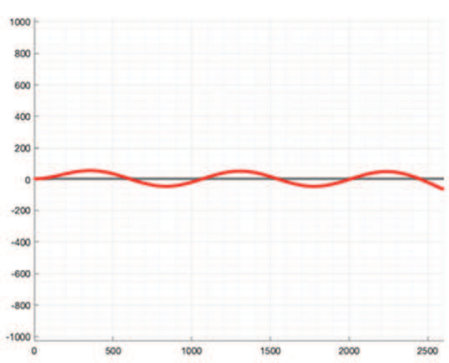

(f)

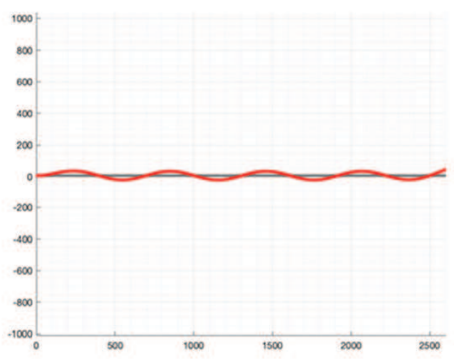

(i)

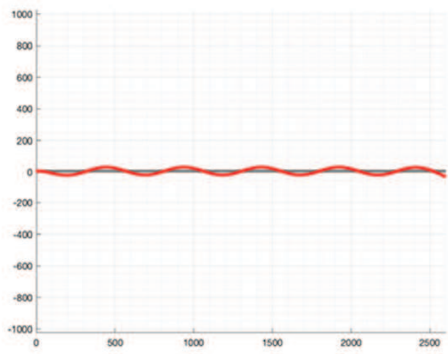

(l)

Figure 2. First 12 natural modes and periods of the p-beam: (a) Ist mode, $T_{1}=19.7 \mathrm{~s}$; (b) $2 \mathrm{nd}$ mode, $T_{2}=3.14 \mathrm{~s}$; (c) $3 \mathrm{rd}$ mode, $T_{3}=1.12 \mathrm{~s}$; (d) 4 th mode, $T_{4}=0.572 \mathrm{~s}$; (e) 5 th mode, $T_{5}=0.166 \mathrm{~s}$; (f) 6 th mode, $T_{6}=0.125 \mathrm{~s} ;(\mathrm{g}) 7$ th mode, $T_{7}=0.0970 \mathrm{~s}$; (h) 8th mode, $T_{8}=0.0888 \mathrm{~s}$; (i) 9th mode, $T_{9}=0.0777 \mathrm{~s}$; (j) I0th mode, $T_{10}=0.0530 \mathrm{~s}$; (k) II th mode, $T_{11}=0.0449 \mathrm{~s}$; (I) I2th mode, $T_{12}=0.0385 \mathrm{~s}$. Red and gray denote the natural modes and the initial configuration, respectively.

acting on point $C$ becomes zero, the pantographic beam does not further compress uniformly, hence the displacement $u_{C}$ reaches a plateau. At this point, a longitudinal (non-uniform) stretch profile starts to propagate from the right extreme of the system, see Figure 5.

The observed dynamics can be thus subdivided neatly in two phases, i.e., the initial uniform compression followed by the compressive stretch profile propagation. Initially, the propagating stretch profile has compact support. Upon reaching the left boundary of the system, it bounces back and, when it returns to the right boundary, a seemingly uniform extension is observed: the displacement $u_{C}$ grows until reaching a positive plateau, which corresponds to a second stretch profile propagation. Eventually, such a profile reaches again, owing to 


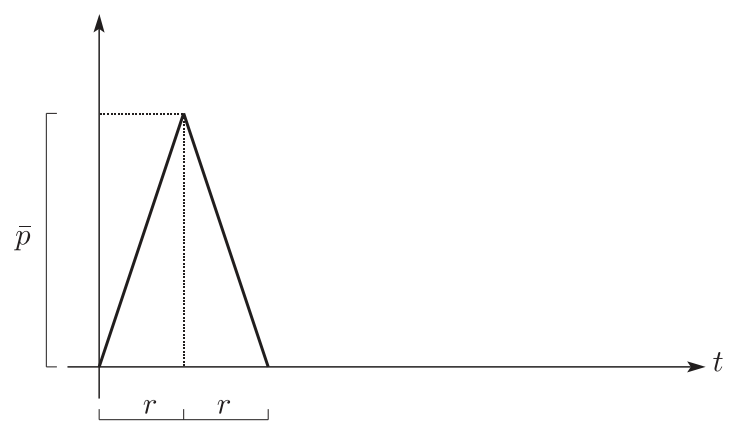

Figure 3. Triangle-shaped impulse having duration and amplitude equal to $2 r$ and $\bar{p}$, respectively.

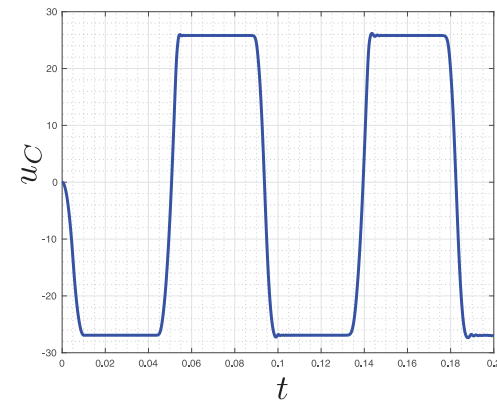

(a)

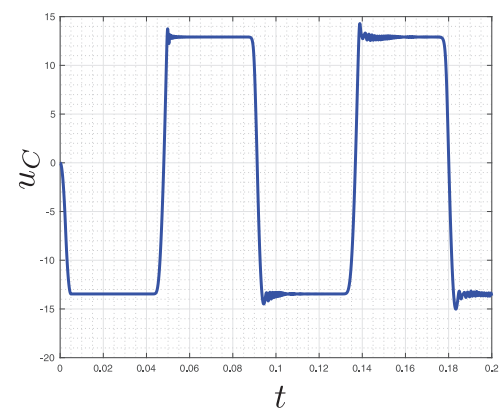

(d)

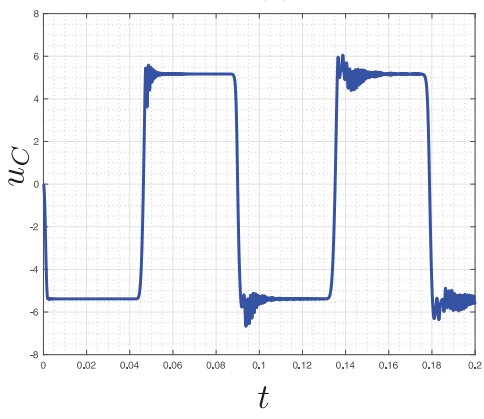

(g)

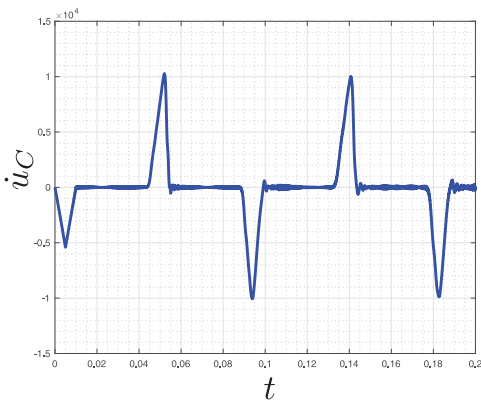

(b)

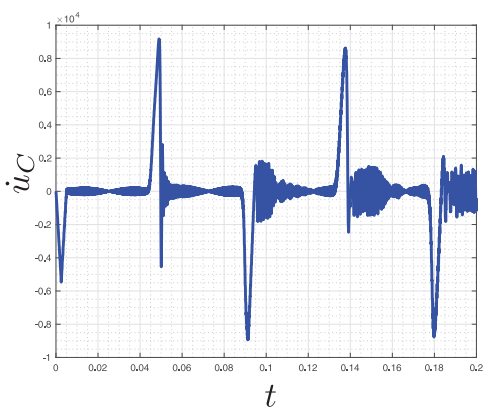

(e)

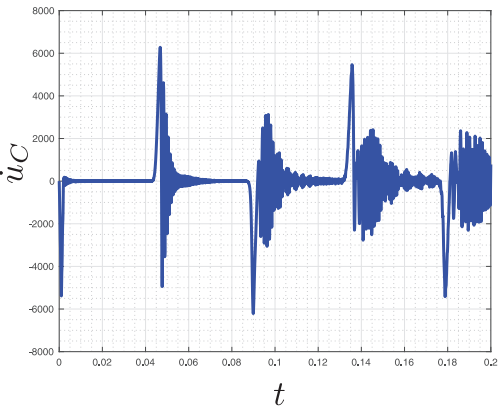

(h)

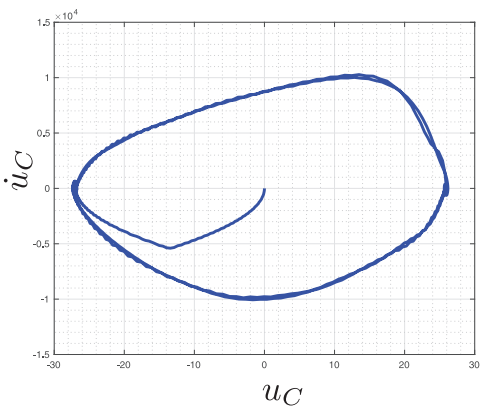

(c)

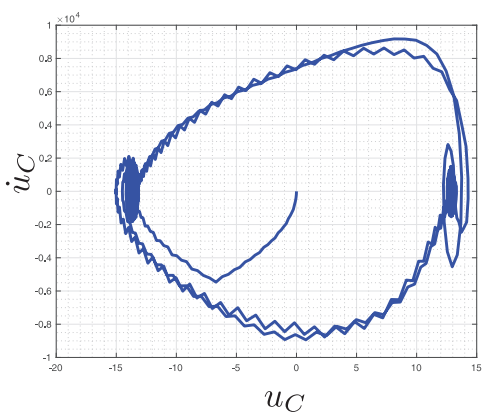

(f)

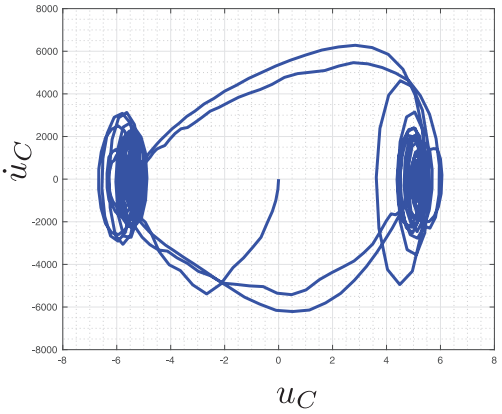

(i)

Figure 4. Hammer test for a pantographic beam with 200 cells and triangle-shaped impulse. Longitudinal displacement (left column) and velocity (middle column) evolutions, and corresponding phase portrait (right column) for the central point of the right end cell, i.e., node $C$. Considered durations of the impulse are (a)-(c) $2 r=0.01 \mathrm{~s}$, (d)-(f) $2 r=0.005 \mathrm{~s}$, and (g)-(i) $2 r=0.002 \mathrm{~s}$.

reflection, the right boundary and the displacement $u_{C}$ decreases until reaching zero. Then, the evolution of $u_{C}$ repeats periodically.

It is worth noting that the time derivative of the displacement $u_{C}$ is zero during the propagation of the stretch profile, whereas it is non-zero otherwise. It is also noted that the form of $\dot{u}_{C}$ resembles the impulse when it is non-zero. Note that, looking at the evolution of the displacement $u_{C}$, rather surprisingly, it seems that nonlinear 


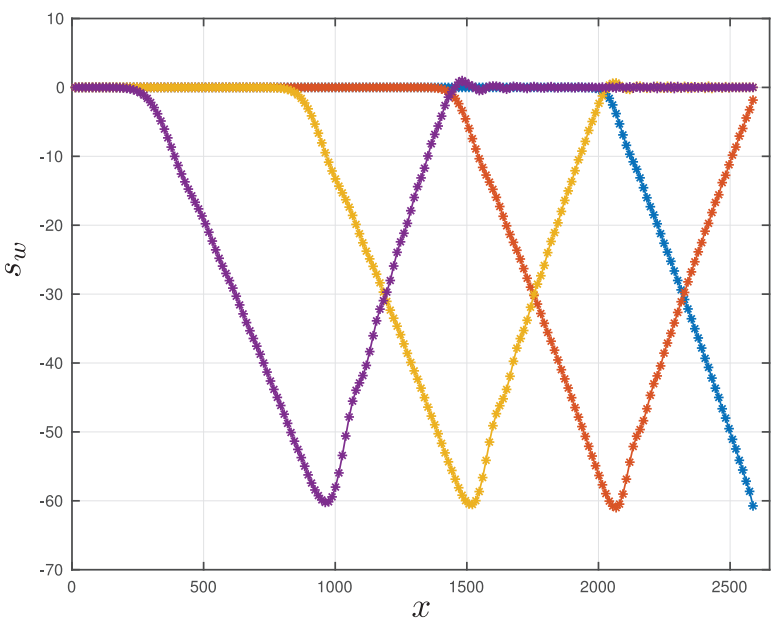

(a)

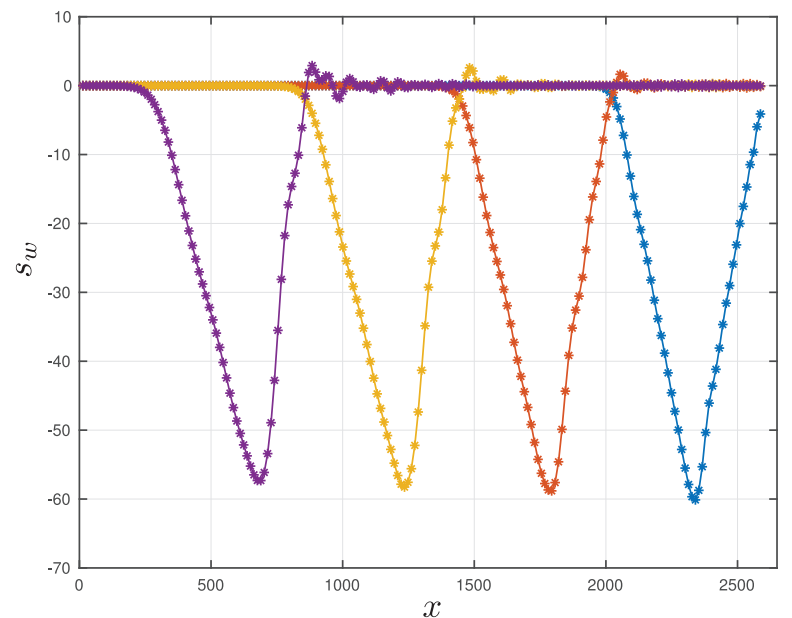

(b)

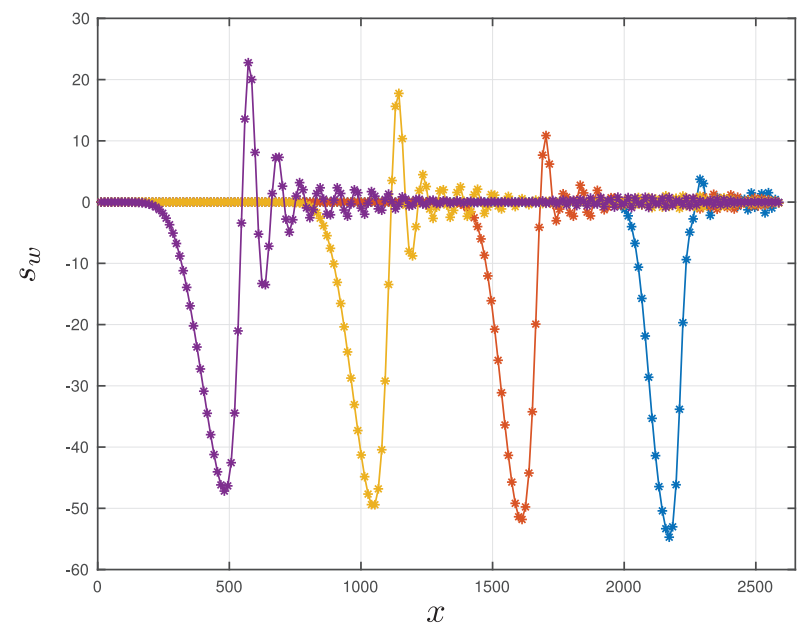

(c)

Figure 5. Hammer test for a p-beam with 200 unit cells and triangle-shaped impulse. Stroboscopic plots of the stretch profile, for (a) $2 r=0.01 \mathrm{~s}$, (b) $2 r=0.005 \mathrm{~s}$, and (c) $2 r=0.002 \mathrm{~s}$, at time instants $t=0.005 \mathrm{~s}$ (cyan), $0.01 \mathrm{~s}$ (red), $0.015 \mathrm{~s}$ (yellow) and $0.02 \mathrm{~s}$ (violet, $\Delta t=10^{-4} \mathrm{~s}$ ). Stretch profiles are multiplied by a factor 100 to make the pictures more readable.

pantographic beams, in longitudinal wave regimes, behave as a kind of nonlinear converter, transforming a concentrated impulse of different forms (see also the results of the simulations presented in Figures 11, 12, 15, and 18 into large-amplitude square waves.

There is another issue that deserves to be addressed, namely the quantification of energy which is lost by the system as a result of numerical errors. Time integration schemes that do not enforce conservation of energy, but only solve balance equations approximately, accumulate small errors that for large times could lead to solutions which are very far from the real one. As the properties, potentialities, and limits of the time integration schemes used in this study are largely unexplored, assessing a posteriori to what extent conservation of energy is fulfilled, because it is not enforced a priori, would provide important information about the scheme. To this end, the total energy, i.e., the sum of the total deformation energy and the total kinetic energy, has been plotted for each time instant in Figure 6. Such a computation is reported only for one exemplary case, i.e., the hammer test with triangle-shaped force impulse, see Figure 3. This figure shows that the total energy initially increases, this being clearly due to the initial force impulse. After the duration of the impulse, no more energy is supplied into within the system and, therefore, the total energy should remain constant in time. Figure 6 shows instead a slight, yet appreciable, decrease, which should however be considered physiological and completely acceptable for the scopes of the analyses presented in this paper. 


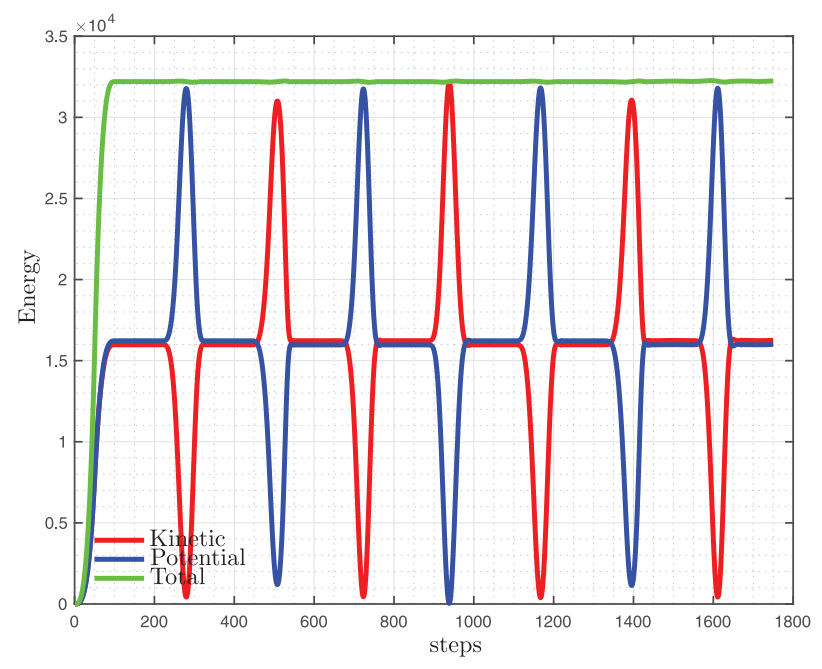

Figure 6. Pantographic beam with 200 cells: evolution of kinetic, potential, and total energies for the hammer test with triangleshaped force impulse.

Looking at Figure 4 it is clearly seen that, when $2 r$ is decreased, some disturbances appear in plots of the displacement $u_{B}$ and its time derivative. These disturbances are due, most likely, to the shape change of the stretch profile during propagation, which can be observed in Figure 5. When $2 r$ is decreased while keeping fixed the total number of cells along with their size, the ratio between $2 r$, i.e., the support of the impulse, and the total length of the pantographic beam decreases. As shown by Figure 5, this entails that the propagating stretch profile changes its shape during propagation. More precisely, for a given time, the stretch profile is increasingly different from its initial shape when $2 r$ decreases. For a given $2 r$, the modified propagating stretch profile is accompanied by an increasingly wide and broad oscillating tail, whereas the amplitude of its initial peak decreases. This shape change indicates that the propagating stretch profile is not, strictly speaking, a traveling wave. Namely, the stretch is not a function of the phase, $k x+\omega t$, with $k$ the wave number, $x$ the space abscissa of mid-points in the reference configuration, $\omega$ the pulsation, when no interaction with boundaries is taking place. On the other hand, it is known that, in the large-deformation regime, existence of traveling wave solutions is generally not granted and, most likely, does not hold. It is worth remarking that deformations experienced by elastic elements within the systems are large in the considered tests, as each time step of the time-integration scheme required many steps to converge, whereas in the small-deformation regime one iteration should be ideally sufficient to reach convergence.

Note also that the presented simulations seem to indicate an interesting nonlinear longitudinal stretch wave propagation property: when $2 r$ is large enough compared with the cell size, the imposed impulse seems to generate a traveling stretch wave, at least for sufficiently short time intervals. On the other hand, when $2 r$ is comparable with the cell size, one can observe, even after short time intervals, a relevant stretch profile variation. Aimed at verifying that to observe changes in the stretch profile during propagation one should let the system evolve for larger times when large $2 r$ are considered, the total size of the pantographic beam has been augmented by considering 1000 cells while keeping the size of each cell and stiffness values unchanged. The hammer test was then performed on the pantographic beam, using as impulse duration $2 r=0.01 \mathrm{~s}$ and as impulse amplitude $\bar{p}=-1000 \mathrm{~N}$. Obviously, for this system, natural periods and modes are different from those reported above for 200 cells and range from $T_{1}=321.3967 \mathrm{~s}$ to $T_{n}=3.3142 \times 10^{-5} \mathrm{~s}$. The lowest purely extensional mode is the $21 \mathrm{st}$, with period $T_{21}=0.34159 \mathrm{~s}$. Based on this evidence, the time step length has been chosen to be $\Delta t=0.001 \mathrm{~s}$. Figure 7(a) reports stroboscopically the stretch profile for five time instants, i.e., $t=0.009 \mathrm{~s}$ (cyan), $0.029 \mathrm{~s}$ (red), $0.049 \mathrm{~s}$ (yellow), $0.069 \mathrm{~s}$ (violet), and $0.089 \mathrm{~s}$ (green). Once again, stretch is amplified by a factor 100 to make the plot more readable. Figure 7(a) suggests that the stretch wave profile is significantly varying during propagation even for larger impulse durations $2 r$, provided that sufficiently long, compared with a given cell size, pantographic beams are considered. It is therefore clear that, while the existence of propagating longitudinal waves seems likely in pantographic beams, more investigations are required to determine the wave profiles generating traveling waves: in fact, after a sufficiently long time interval, the impulse applied on the 


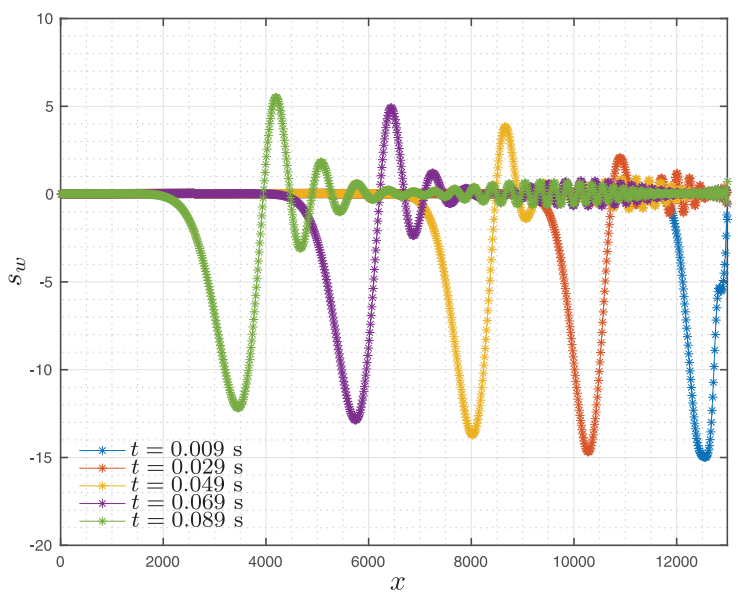

(a)

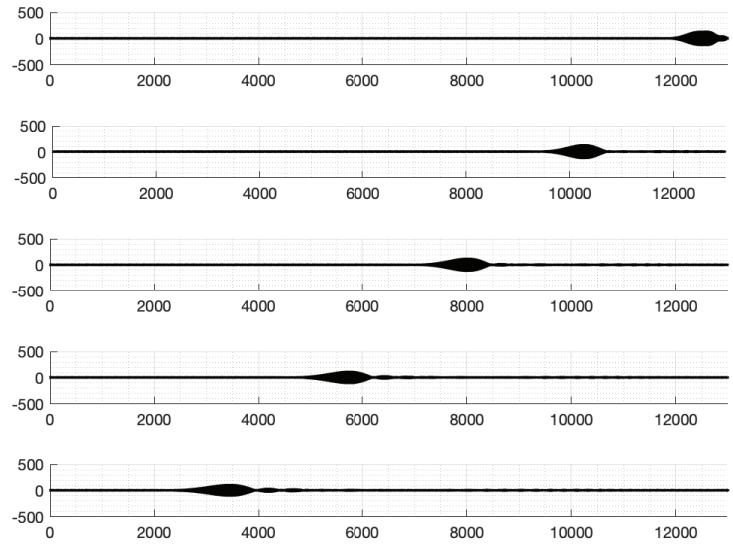

(b)

Figure 7. Hammer test for a p-beam with 1000 unit cells and triangle-shaped impulse: (a) evolution of the stretch profile; (b) evolution of the current shape. Stroboscopic plots of the stretch profile and of the current shape, for $2 r=0.01 \mathrm{~s}$, at time instants $t=0.009,0.029,0.049,0.069$, and $0.089 \mathrm{~s}$. Stretch profiles and transversal displacements are multiplied by factors of 100 and 2000 , respectively, to make the pictures more readable.

right end of the pantographic beam decomposes in a series of waves, some of which may be traveling waves. In this context, some further investigations may be necessary.

Aimed at giving a more complete picture of the obtained results, Figure 8 shows stroboscopically the current shape of the pantographic beam made up of 200 cells at time instants, from top to bottom, $t=0.005,0.002$, $0.011,0.016$, and $0.021 \mathrm{~s}$, being the employed duration of the impulse $2 r=0.01 \mathrm{~s}$. Transversal displacement is multiplied by a scale factor of 500 to make the pictures more readable. Analogously, Figure 7(b) shows stroboscopically the current shape of the pantographic beam made up of 1000 cells at time instants, from top to bottom, $t=0.009,0.029,0.049,0.069$, and $0.089 \mathrm{~s}$. Transversal displacement is multiplied by a scale factor of 2000 to make the pictures more readable.

The hammer test was performed numerically also making use of compressive impulsive loads different from the triangle-shaped load discussed so far. More specifically, trapezoid-shaped and zig-zag-shaped loads were considered for the pantographic beam with 200 cells, see Figures 9 and 10, respectively.

Figures 11 and 12 report, for the hammer test with trapezoid-shaped and zig-zag-shaped impulse, respectively, the longitudinal displacement (left) and velocity (center) evolutions, together with the corresponding phase portrait (right), for the right end cell midpoint, i.e., node $C$. The impulse was set in both cases equal to $2 r=0.01 \mathrm{~s}$. Comments on these figures are analogous to those made for the same test when the impulse function was triangle-shaped.

We now delve more deeply into the reason why the zig-zag-shaped impulse function was considered. As is noticeable when looking at Figures 5 and 7, when subjecting the clamped pantographic beam to a compressive triangle-shaped impulsive load acting on its right end, the stretch profile, which is initially triangle-shaped, changes while propagating. Indeed, the initial peak lowers its magnitude, whereas a smaller peak arises and progressively increases in magnitude. A kind of oscillating tail is also forming. Seemingly, a redistribution of the strain energy along the beam axis occurs during the stretch profile propagation. A legitimate question concerns whether this redistribution leads to a stationary, i.e., persistent, propagating profile. Aimed at checking whether the modified profile is (close to be) persistent or not, a compression force impulse, i.e., the zig-zag-shaped impulse, shaped similarly, albeit not equally, to the modified stretch profile has been applied at the right end.

Figure 13 reports stroboscopically the stretch profile for five time instants, i.e., $t=0.009 \mathrm{~s}$ (cyan), $t=$ $0.029 \mathrm{~s}$ (red), $t=0.049 \mathrm{~s}$ (yellow), $t=0.069 \mathrm{~s}$ (violet), and $t=0.089 \mathrm{~s}$ (green). The stretch $s_{w}$ is amplified by a factor of 100 to make the plots more readable. Analogously to what we have observed previously for the triangleshaped impulse, also for the zig-zag-shaped impulse function $2 r=0.01 \mathrm{~s}$ is too large with respect to the total length of the system to appreciate possible changes in the stretch profile during propagation. Therefore, as done for the triangle-shaped impulse, the total size of the pantographic beam has been augmented by considering 

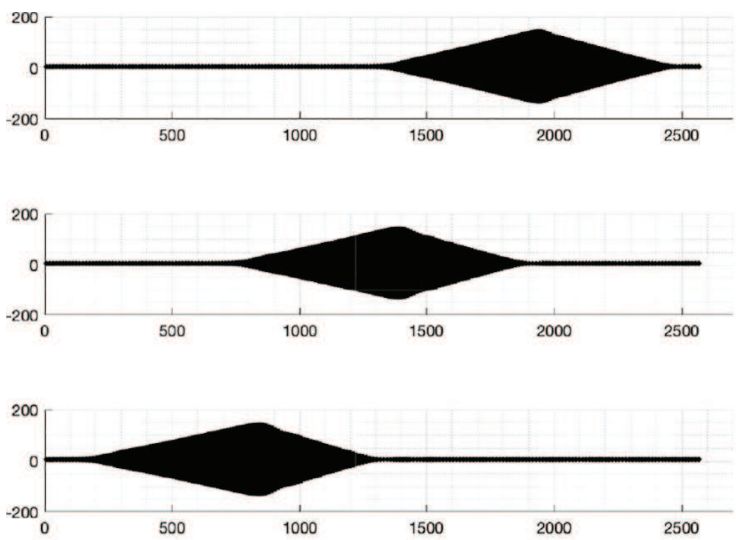

(a)
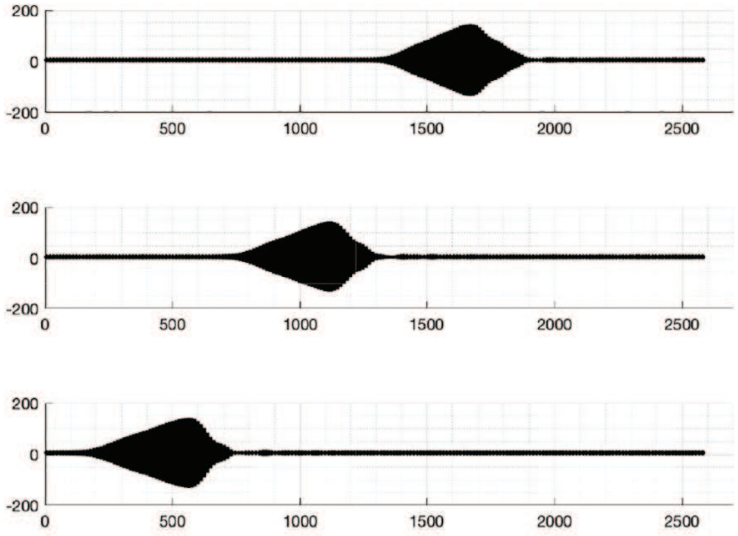

(b)
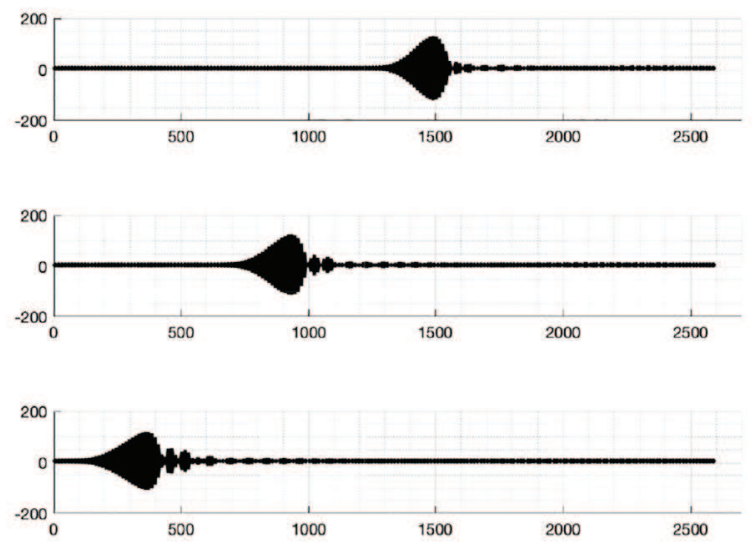

(c)

Figure 8. Hammer test for a p-beam with 200 unit cells and triangle-shaped impulse. Stroboscopic plots of the current shape, for (a) $2 r=0.01 \mathrm{~s}$, (b) $2 r=0.005 \mathrm{~s}$, and (c) $2 r=0.002 \mathrm{~s}$, at time instants $t=0.011 \mathrm{~s}, t=0.016 \mathrm{~s}$ and $t=0.021 \mathrm{~s}$. Transversal displacements are multiplied by a factor 500 to make the pictures more readable.

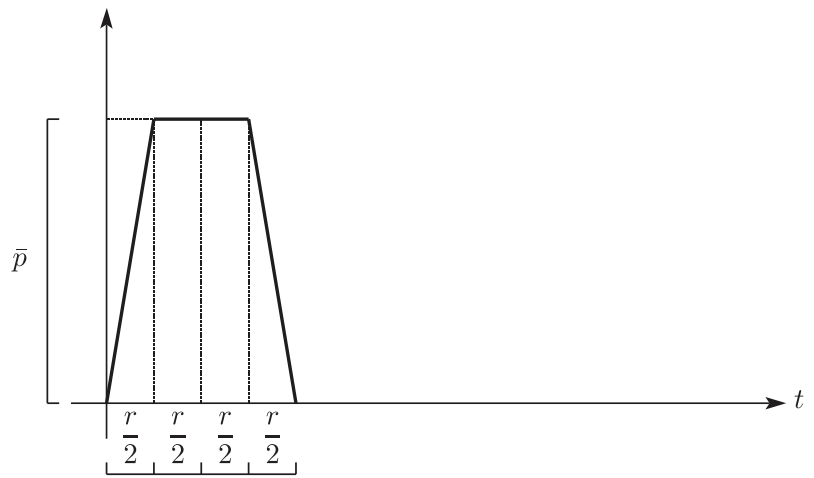

Figure 9. Trapezoid-shaped impulse having duration and amplitude equal to $2 r$ and $\bar{p}$, respectively.

1000 cells while keeping the size of each cell unchanged. The same test, i.e., the hammer test with zig-zagshaped impulse, was then performed on the pantographic beam, using as impulse duration $2 r=0.01 \mathrm{~s}$ and as impulse amplitude $\bar{p}=-1000 \mathrm{~N}$. As mentioned previously, for this system natural periods and modes are different from those computed above in the case of 200 cells and, accordingly, the time step length has been chosen to be $\Delta t=0.001 \mathrm{~s}$. 


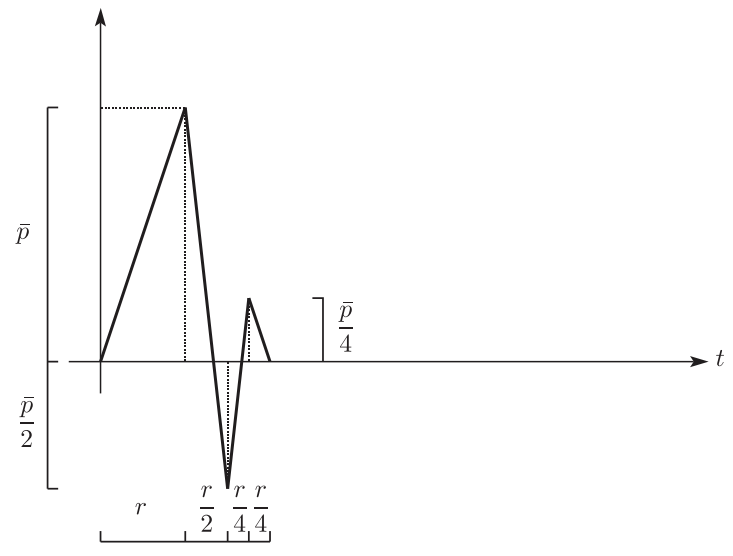

Figure 10. Zig-zag-shaped impulse.

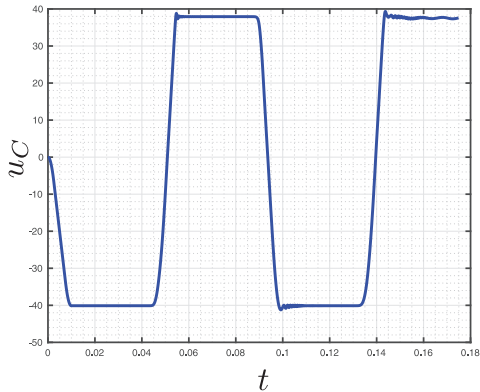

(a)

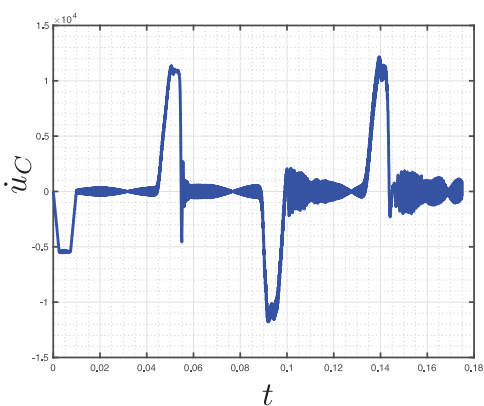

(b)

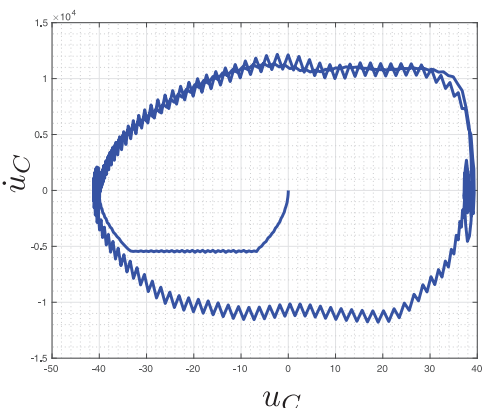

(c)

Figure II. Hammer test for a pantographic beam with 200 cells and trapezoid-shaped impulse. Longitudinal displacement $u_{C}$ (a) and velocity $\dot{u}_{C}$ (b) evolutions, and corresponding phase portrait for the displacement $u_{C}$ (c) for the central point of the right end cell, i.e., node $C$. The considered duration of the impulse is $2 r=0.01 \mathrm{~s}\left(\Delta t=10^{-4} \mathrm{~s}\right)$.

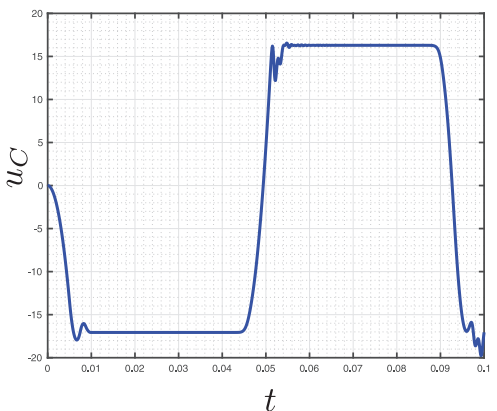

(a)

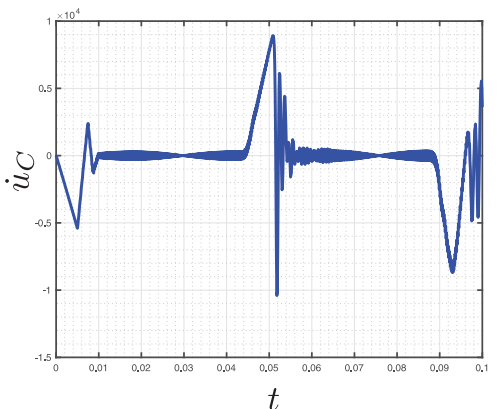

(b)

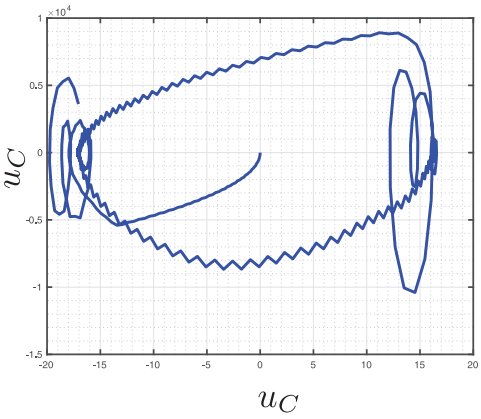

(c)

Figure 12. Hammer test for a pantographic beam with 200 cells and zig-zag-shaped impulse. Longitudinal displacement $u_{C}$ (a) and velocity $\dot{u}_{C}$ (b) evolutions, and corresponding phase portrait for the displacement $u_{C}$ (c) for the central point of the right end cell, i.e. node $C$. The considered duration of the impulse is $2 r=0.01 \mathrm{~s}\left(\Delta t=10^{-4} \mathrm{~s}\right)$.

Figure 14 reports stroboscopically the stretch profile for five time instants, i.e., $t=0.02 \mathrm{~s}$ (cyan), $t=0.04 \mathrm{~s}$ (red), $t=0.06 \mathrm{~s}$ (yellow), $t=0.08 \mathrm{~s}$ (violet), and $t=0.1 \mathrm{~s}$ (green). The stretch $s_{w}$ is amplified by a factor of 100 to make the plots more readable. Figure 14 suggests that, as for the hammer test with triangle-shaped impulse, the stretch profile is significantly varying during propagation provided that sufficiently long pantographic beams are considered for a given cell size. Evidence collected so far, while suggesting that the problem of finding a persistent propagating stretch profile generated by an impulse loading is difficult, does not provide however a definitive answer on the existence of such stretch profiles. Nevertheless, we believe that the previously exposed ideas underlying the use of the zig-zag-shaped impulse should guide future investigations. Such 


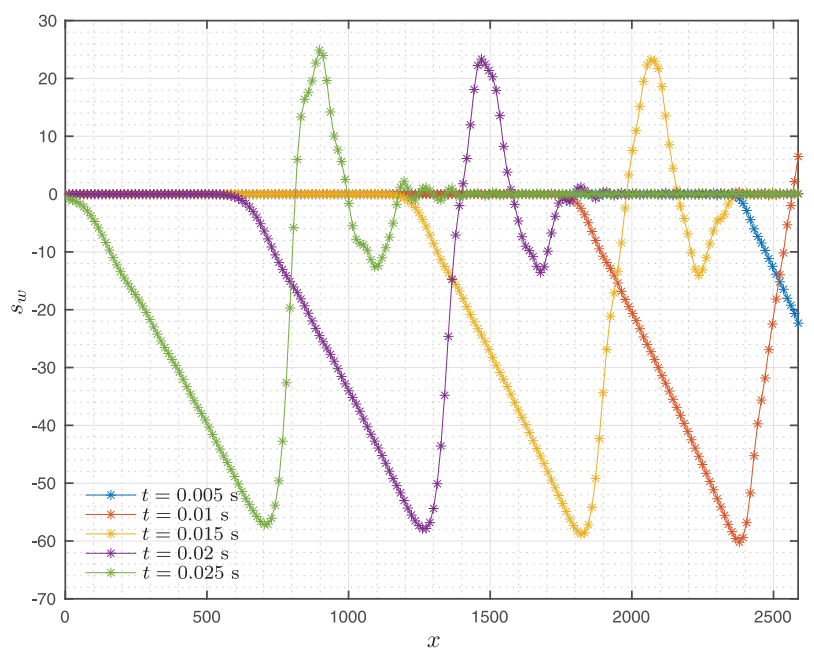

Figure 13. Hammer test for a p-beam with 200 unit cells and zig-zag-shaped impulse. Stroboscopic plots of the stretch profile, for $2 r=0.01 \mathrm{~s}$, at time instants $t=0.005 \mathrm{~s} \mathrm{(cyan),} t=0.01 \mathrm{~s}$ (red), $t=0.015 \mathrm{~s}$ (yellow), $t=0.02 \mathrm{~s}$ (violet), and $t=0.025 \mathrm{~s}$ (green, $\left.\Delta t=10^{-4} \mathrm{~s}\right)$. Stretch profiles are multiplied by a factor of 100 to make the pictures more readable.

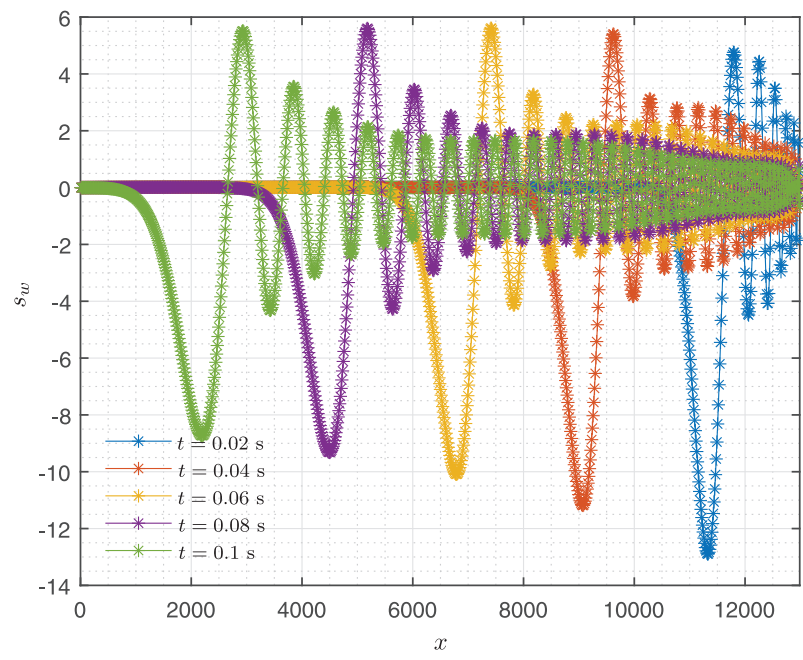

Figure 14. Hammer test for a p-beam with 1000 unit cells and zig-zag-shaped impulse. Stroboscopic plots of the stretch profile, for $2 r=0.01 \mathrm{~s}$, at time instants $t=0.02 \mathrm{~s}$ (cyan), $t=0.04 \mathrm{~s}$ (red), $t=0.06 \mathrm{~s}$ (yellow), $t=0.08 \mathrm{~s}$ (violet), and $t=0.1 \mathrm{~s}$ (green, $\Delta t=10^{-3} \mathrm{~s}$ ). Stretch profiles are multiplied by a factor of 100 to make the pictures more readable.

investigations might involve parametric studies on the features that characterize the zig-zag-shaped impulse, such as the support of each triangle-shaped component of such a zig-zag, the amplitude of the peaks, etc.

\subsection{Jerk test}

What we call jerk test is very close to the hammer test with triangle-shaped impulse described in the foregoing, except for the fact that, in this case, the impulse is a traction force $(\bar{p}=4000 \mathrm{~N})$ applied on the central point of the right end cell, i.e., node $C$. Parameters of the time-integration scheme were set equal to those utilized for the hammer test performed on a pantographic beam with 200 cells. In particular, the time step was chosen equal to $\Delta t=10^{-4} \mathrm{~s}$. Figure 15 reports the longitudinal displacement (left) and velocity (center) evolutions, together with the corresponding phase portrait (right), for the right end cell midpoint, i.e., node $C$. The impulse was set equal to $2 r=0.01 \mathrm{~s}, 2 r=0.005 \mathrm{~s}$, and $2 r=0.002 \mathrm{~s}$. Comments on this figure are analogous to those made for the same test when the impulse function was triangle-shaped and compressive. 


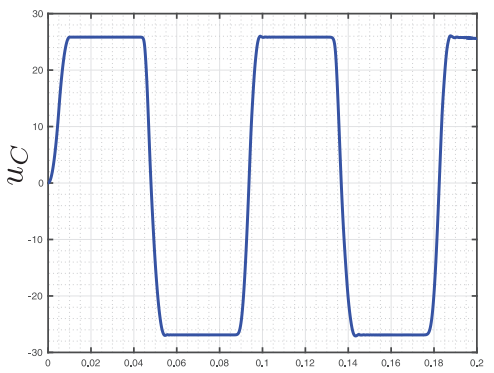

$t$

(a)

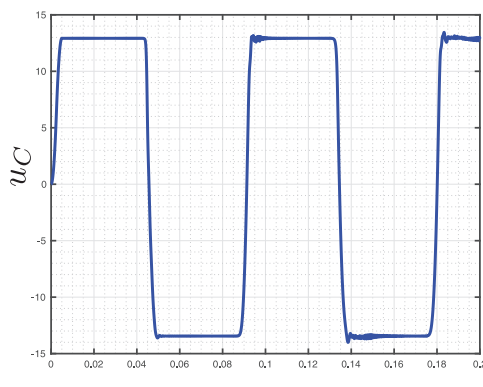

$t$

(d)

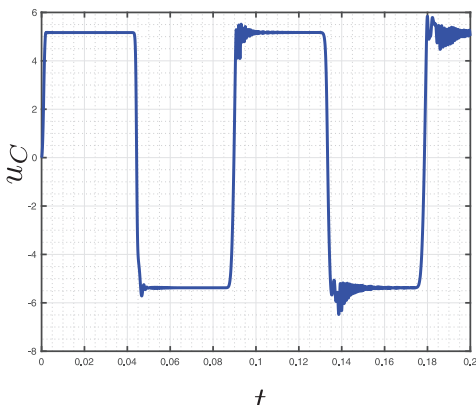

(g)

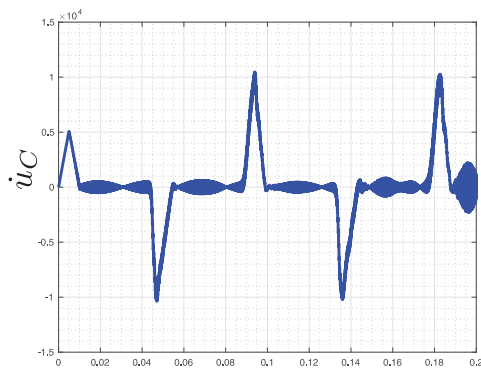

$t$

(b)

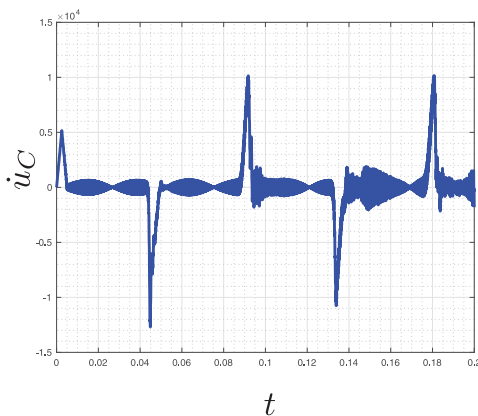

(e)

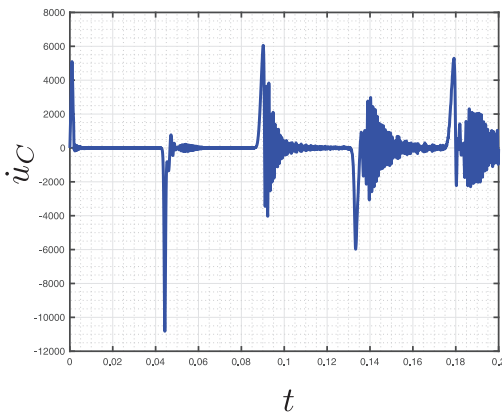

(h)

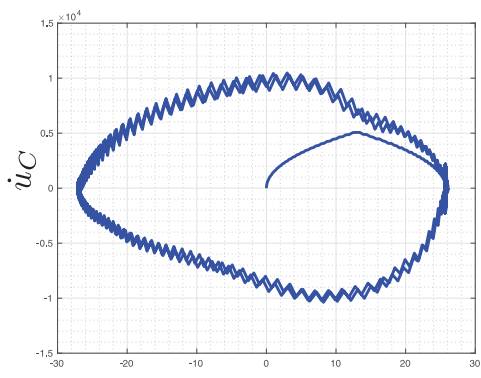

$u_{C}$

(c)

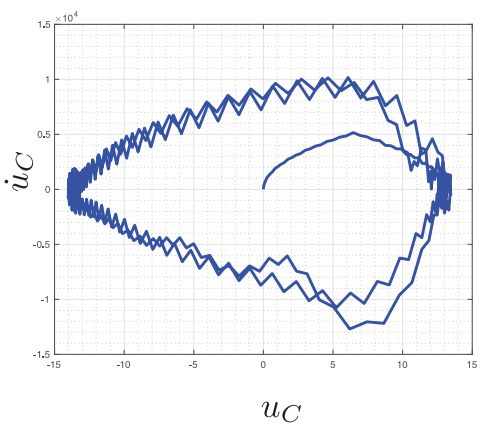

(f)

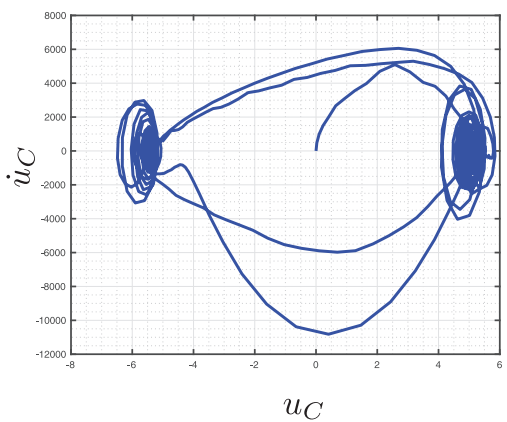

(i)

Figure I5. Jerk test for a pantographic beam with 200 cells and triangle-shaped impulse. Longitudinal displacement (left column) and velocity (middle column) evolutions, and corresponding phase portrait (right column) for the central point of the right end cell, i.e., node $C$. Considered durations of the impulse are (a)-(c) $2 r=0.01 \mathrm{~s}$, (d) $-(\mathrm{f}) 2 r=0.005 \mathrm{~s}$, and (g) - (i) $2 r=0.002 \mathrm{~s}$.

Figure 16 reports stroboscopically, for different durations of the impulse, i.e., $2 r=0.01,0.005$, and $0.002 \mathrm{~s}$, the longitudinal strain profile for four time instants, i.e., $t=0.005 \mathrm{~s}$ (cyan), $t=0.01 \mathrm{~s}$ (red), $t=0.015 \mathrm{~s}$ (yellow), and $t=0.02 \mathrm{~s}$ (violet). The stretch $s_{w}$ is amplified by a factor of 100 to make the plots more readable.

Figure 17 shows stroboscopically the current shape of the pantographic beam made up of 200 cells at time instants, from top to bottom, for $t=0.011,0.016$, and $0.021 \mathrm{~s}$, with the employed duration of the impulse being $2 r=0.01,0.005$ and $0.002 \mathrm{~s}$. Once again, transversal displacements are multiplied by a scale factor of 500 to make the pictures more readable.

\subsection{Double hammer test}

In this test, the pantographic beam made up of 200 cells is subjected to the following kinematic constraints $v_{A}=u_{D}=u_{E}=v_{C}=0$, with $D$ and $E$ being the nodes of the p-beam lying on its vertical symmetry axis and having, respectively, coordinates $(L / 2,0)$ and $(L / 2, f)$, see Figure 1. Essentially, two horizontal rollers are applied at central points of both left and right end cells. In addition, vertical rollers are applied to the upper 


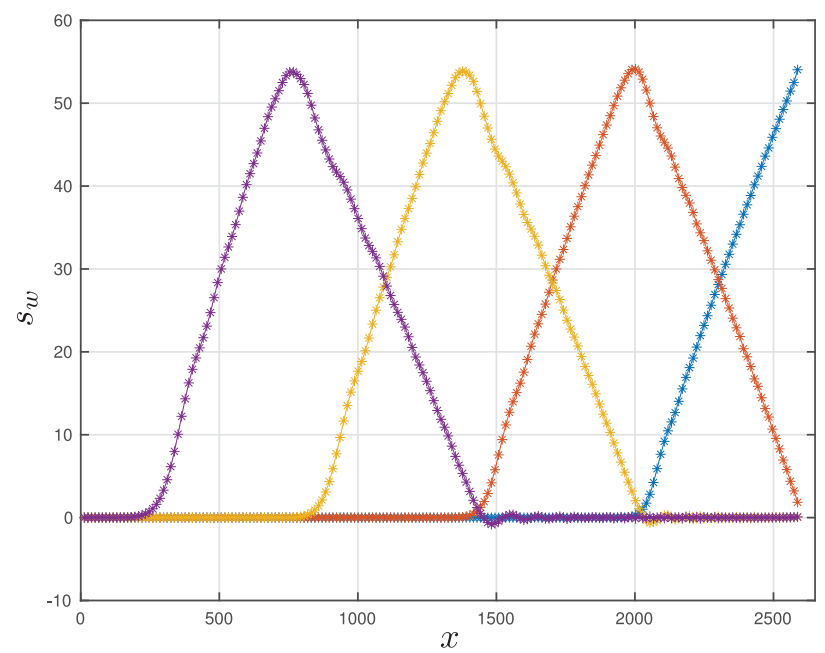

(a)

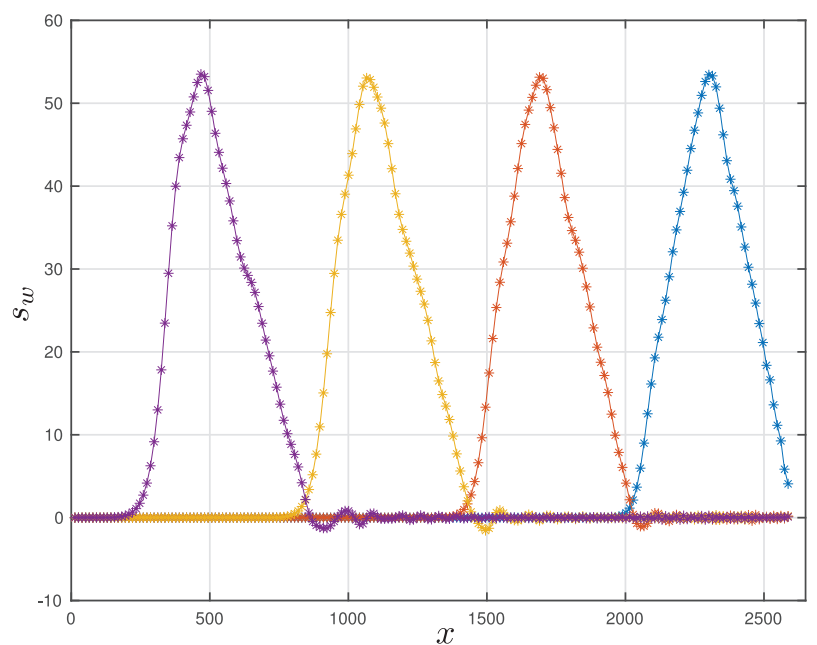

(b)

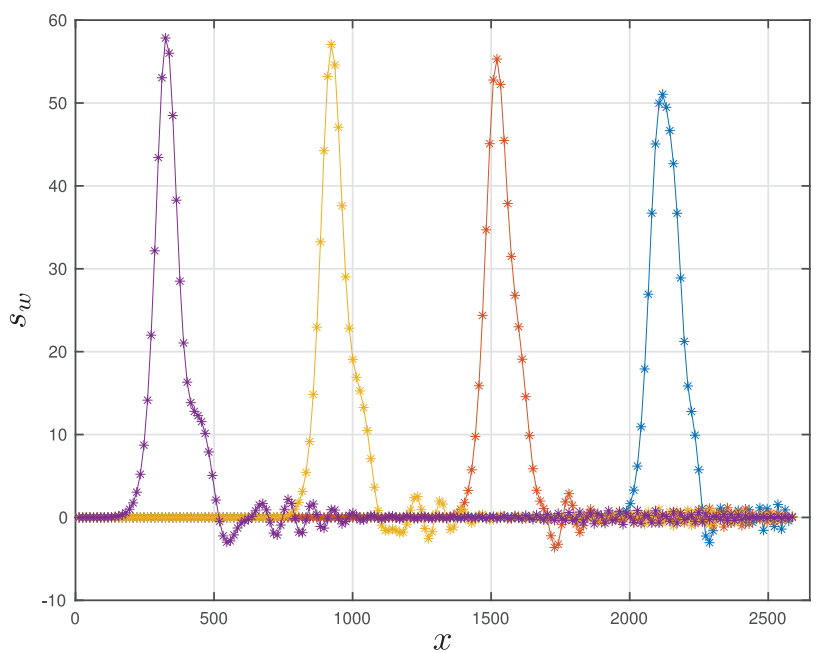

(c)

Figure 16. Jerk test for a p-beam with 200 unit cells and triangle-shaped impulse. Stroboscopic plots of the stretch profile, for (a) $2 r=0.01 \mathrm{~s}$, (b) $2 r=0.005 \mathrm{~s}$, and (c) $2 r=0.002 \mathrm{~s}$, at time instants $t=0.005 \mathrm{~s}$ (cyan), $t=0.01 \mathrm{~s}$ (red), $t=0.15 \mathrm{~s}$ (yellow), and $t=0.2 \mathrm{~s}$ (violet, $\Delta t=10^{-4} \mathrm{~s}$ ). Stretch profiles are multiplied by a factor of 100 to make the pictures more readable.

and lower points of the central cell. These constraints are sufficient to avoid any rigid motion, while being compatible with the symmetry of the sought deformed shape with respect to the vertical and horizontal axes of the beam. Clearly, this change in the constraints affects the natural periods and modes found by means of modal analysis, which are thus different from those computed above in the case of 200 cells for clamped boundary conditions. For these boundary conditions, the period of the lowest purely extensional mode is $T_{13}=0.044307 \mathrm{~s}$, whereas the first and last natural periods are $T_{1}=7 \mathrm{~s}$ and $T_{n}=3.3065 \times 10^{-5} \mathrm{~s}$, respectively. Accordingly, the time step has been set as $\Delta t=10^{-4} \mathrm{~s}$. The quantities $\bar{p}$ and $2 r$ were fixed, respectively, $\bar{p}=4000 \mathrm{~N}$ and $2 r=0.01 \mathrm{~s}$. Figure 18 reports the longitudinal displacement (left) and velocity (center) evolutions, together with the corresponding phase portrait (right), for the central point of the right end cell, i.e., node $C$. Figure 19 reports stroboscopically the stretch profile for five time instants, i.e., $t=0.005 \mathrm{~s}$ (cyan), $t=0.01 \mathrm{~s}$ (red), $t=0.015 \mathrm{~s}$ (yellow), $t=0.02 \mathrm{~s}$ (violet), and $t=0.025 \mathrm{~s}$ (green). Stretch $s_{w}$ is amplified by a factor of 100 to make the plots more readable. Figure 20 shows stroboscopically the current shape of the pantographic beam at time instants, from top to bottom, $t=0.005,0.01,0.015$, and 0.02 , and $0.025 \mathrm{~s}$, with the employed duration of the impulse being $2 r=0.01 \mathrm{~s}$. Transversal displacements are multiplied by a scale factor of 250 to make the pictures more readable. 

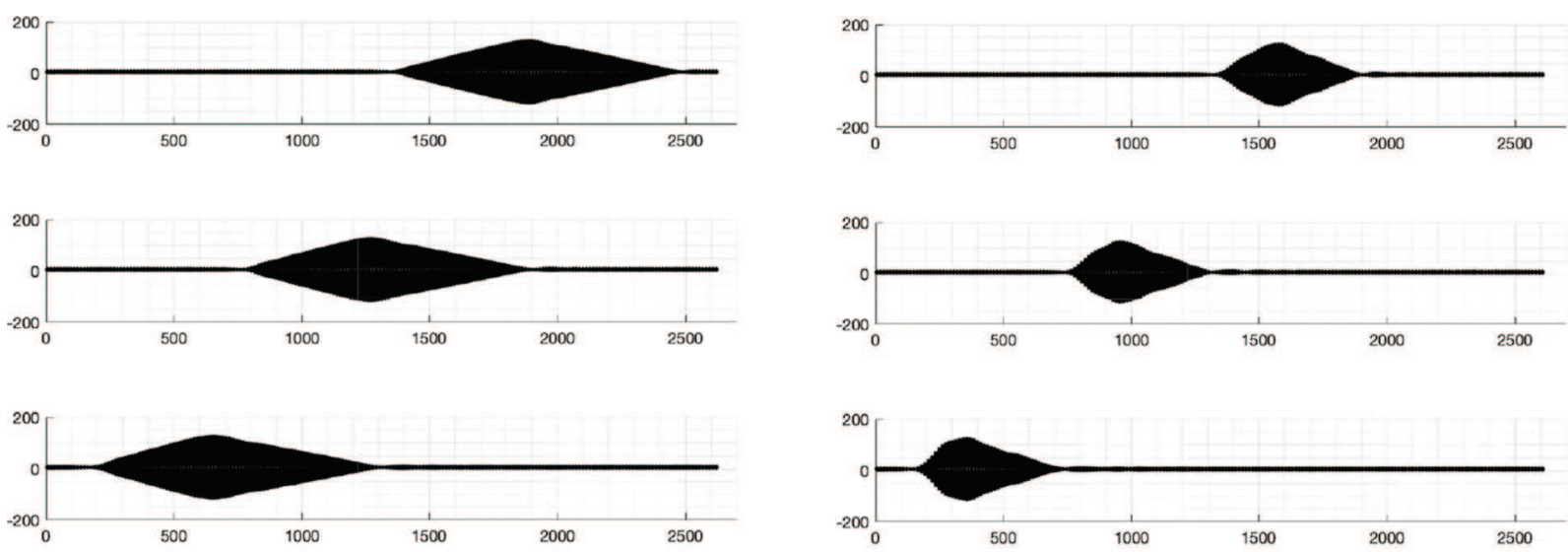

(a)

(b)
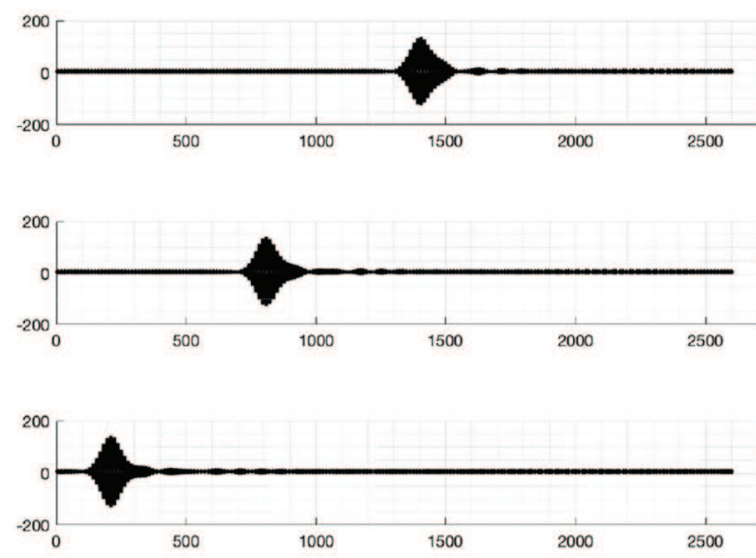

(c)

Figure 17. Hammer test for a p-beam with 200 unit cells and triangle-shaped impulse. Stroboscopic plots of the current shape, for (a) $2 r=0.01 \mathrm{~s}$, (b) $2 r=0.005 \mathrm{~s}$, and (c) $2 r=0.002 \mathrm{~s}$, at time instants $t=0.011,0.015$, and $0.021 \mathrm{~s}$. Transversal displacements are multiplied by a factor of 500 to make the pictures more readable.

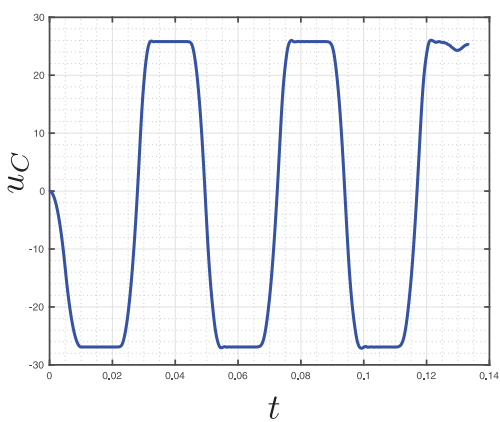

(a)

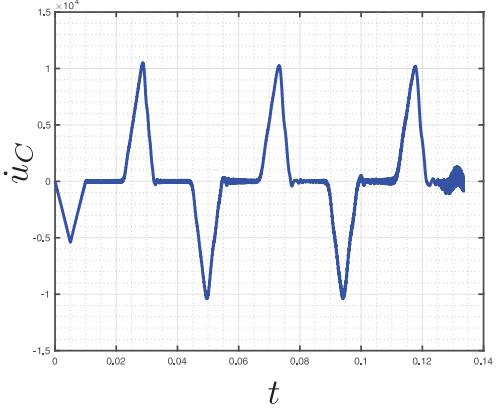

(b)

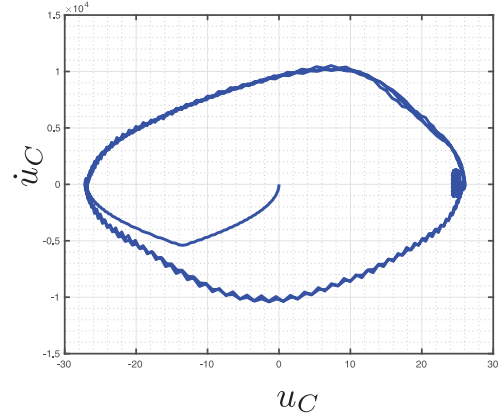

(c)

Figure 18. Double hammer test for a pantographic beam with 200 cells and triangle-shaped impulse. Longitudinal displacement (a) and velocity (b) evolutions, and corresponding phase portrait (c) for the central point of the right end cell, i.e. node $C$. The considered duration of the impulse is $2 r=0.01 \mathrm{~s}\left(\Delta t=10^{-4} \mathrm{~s}\right)$.

\subsection{Hammer test on a pantographic beam with perfect pivots}

The hammer test with triangle-shaped impulse function is considered for a pantographic beam with 200 unit cells. All pivots are assumed to be perfect hinges and, therefore, the stiffness $c$ is set to zero. The stiffnesses 


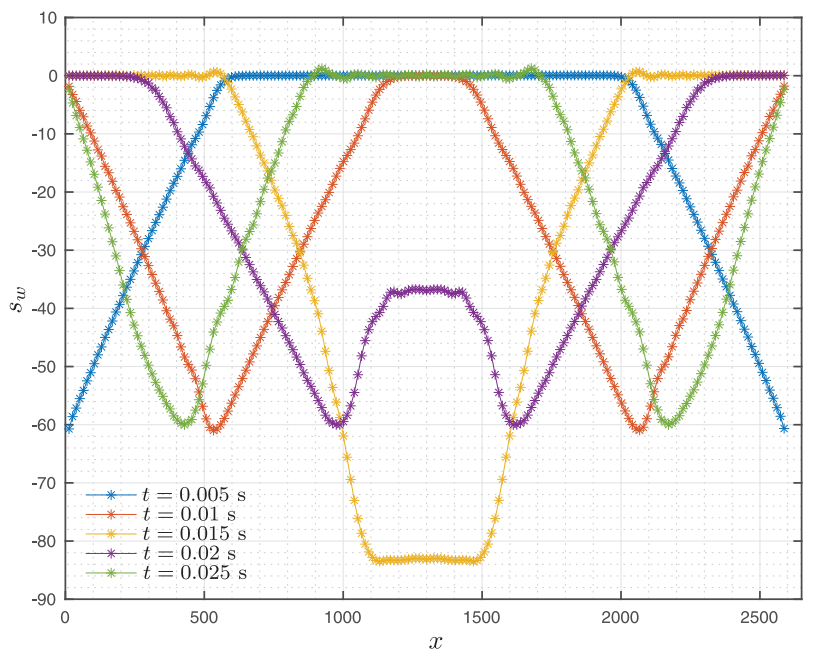

Figure 19. Double hammer test for a p-beam with 200 unit cells and triangle-shaped impulse. Stroboscopic plots of the stretch profile, for $2 r=0.01 \mathrm{~s}$, at time instants $t=0.005 \mathrm{~s}$ (cyan), $t=0.01 \mathrm{~s}$ (red), $t=0.015 \mathrm{~s}$ (yellow), $t=0.02 \mathrm{~s}$ (violet), and $t=0.025 \mathrm{~s}$ (green); $\Delta t=10^{-4} \mathrm{~s}$. Stretch profiles are multiplied by a factor 100 to make the pictures more readable.
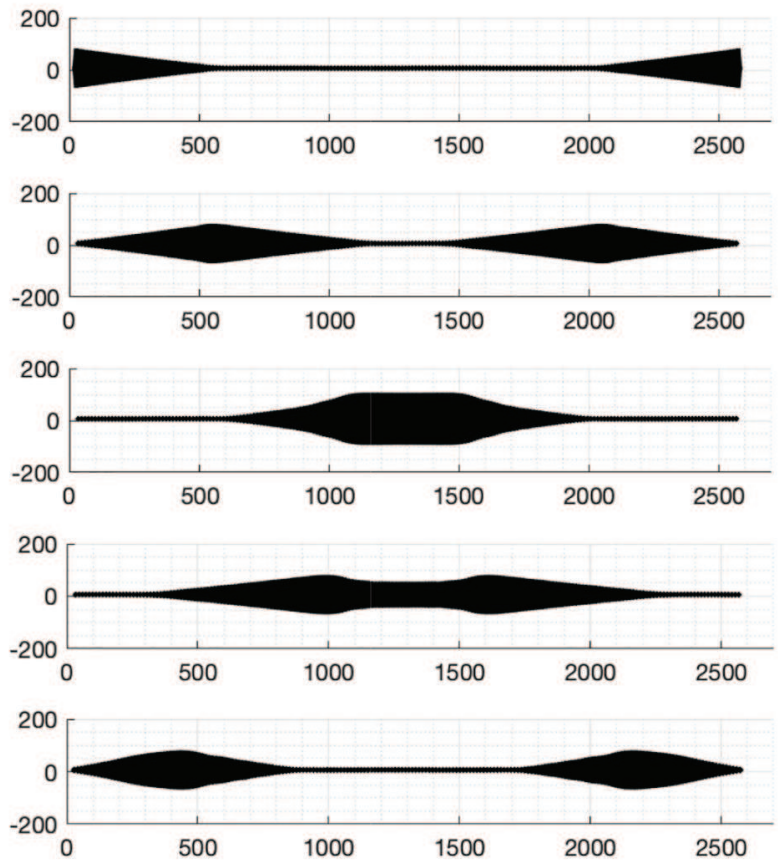

Figure 20. Double hammer test for a p-beam with 200 unit cells and triangle-shaped impulse. Stroboscopic plots of the current shape, for $2 r=0.01 \mathrm{~s}$, at time instants $t=0.005,0.01,0.015,0.02$, and $0.025 \mathrm{~s}\left(\Delta t=10^{-4} \mathrm{~s}\right)$. Transversal displacements are multiplied by a factor of 250 to make plots more readable.

$a$ and $b$ are chosen according to Table 1 . With this change in the stiffness $c$, the system becomes statically undetermined when an axial force, such as the considered impulsive load, is applied to the pantographic beam. Therefore, having in mind to fix the elongation of the mid-line, the constraint $u_{B}=0$ was prescribed. This also corresponds, at the macro-scale, i.e., for the homogenized continuum (see [55]), to applying a (reactive) double force. Clearly, this change in the stiffness $c$ affects the natural periods and modes found by means of the eigenvalue problem to be solved around the initial configuration. The lowest purely extensional mode is the first, which has a period $T_{1}=28.372 \mathrm{~s}$. Accordingly, the time step has been set as $\Delta t=0.05 \mathrm{~s}$. The quantities $\bar{p}$ and $2 r$ were fixed equal to, respectively, $\bar{p}=0.1 \mathrm{~N}$ and $2 r=1 \mathrm{~s}$. Figure 21 reports the longitudinal 


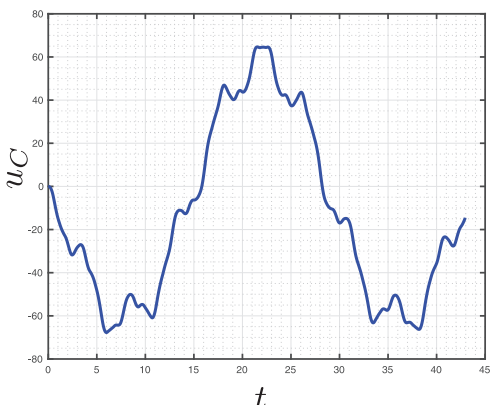

(a) $2 r=1 \mathrm{~s}$

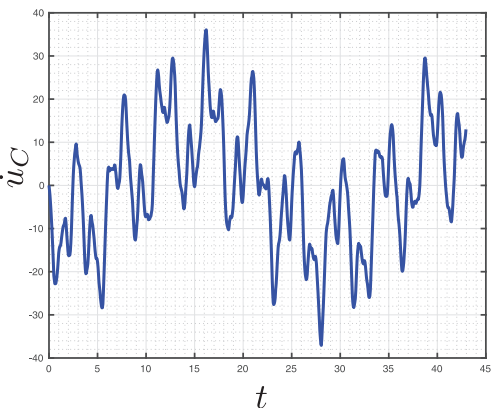

(b) $2 r=1 \mathrm{~s}$

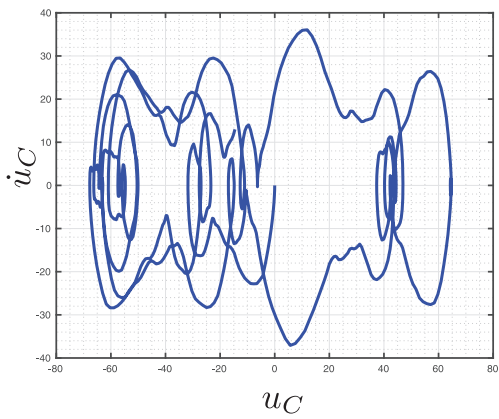

(c) $2 r=1 \mathrm{~s}$

Figure 2 I. Hammer test for a pantographic beam with perfect pivots, 200 cells, and triangle-shaped impulse. Longitudinal displacement (a) and velocity (b) evolutions, and corresponding phase portrait (c) for the central point of the right end cell, i.e., node $C$. The considered duration of the impulse is $2 r=1 \mathrm{~s}(\Delta t=0.05 \mathrm{~s})$.

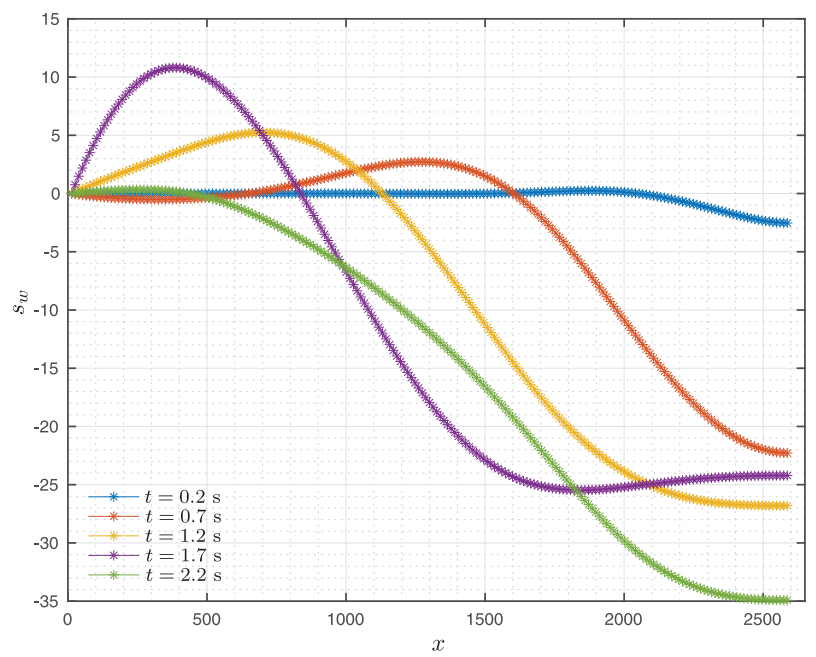

Figure 22. Hammer test for a pantographic beam with perfect pivots, 200 cells, and triangle-shaped impulse. Stroboscopic plots of the stretch profile, for $2 r=1 \mathrm{~s}$, at time instants $t=0.2 \mathrm{~s}$ (cyan), $t=0.7 \mathrm{~s}$ (red), $t=1.2 \mathrm{~s}$ (yellow), $t=1.7 \mathrm{~s}$ (violet), and $t=2.2 \mathrm{~s}$ (green); $\Delta t=0.05 \mathrm{~s}$. Stretch profiles are multiplied by a factor of 100 to make the pictures more readable.

displacement (left) and velocity (center) evolutions, together with the corresponding phase portrait (right), for the central point of the right end cell, i.e., node $C$. Figure 22 reports stroboscopically the stretch profile for four time instants, i.e., $t=0.2 \mathrm{~s}$ (cyan), $t=0.7 \mathrm{~s} \mathrm{(red),} t=1.2 \mathrm{~s}$ (yellow), $t=1.7 \mathrm{~s}$ (violet), and $t=2.2 \mathrm{~s}$ (green). The stretch is amplified by a factor of 100 to make the plots more readable. Figure 23 shows stroboscopically the current shape of the pantographic beam at, from top to bottom, $t=0.2 \mathrm{~s}$ (cyan), $t=0.7 \mathrm{~s}$ (red), $t=1.2 \mathrm{~s}$ (yellow), $t=1.7 \mathrm{~s}$ (violet), and $t=2.2 \mathrm{~s}$ (green). Once again, transversal displacements are multiplied by a scale factor of 500 to make the pictures more readable.

In the case of perfect pivots, the characteristic length scale for the elastic energy of the equivalent continuum, which is related to the bending stiffness of micro-beams, becomes infinite. Therefore, it is not possible to assume that high gradients of displacement are concentrated inside small internal boundary layers. As a consequence, the generation of the square-like wave observed for the displacement $u_{C}$ in the case of non-negligible pivots' stiffness disappears: the elastic deformation energy is never concentrated in a small space interval during wave propagation.

It is worth expanding the previous sentences, as we stress that this point is crucially characterizing the mechanics of pantographic beams and, more generally, pantographic structures. The contribution [63] has recently proven that, for a pantographic beam, shear-rotational springs result at the macro-scale in an additive strain energy density term depending only on the stretch of the centerline. The contribution [55] has instead proven that, when bending-rotational springs are much weaker than extensional springs, which is what we considered in Table 1, then no dependence on the stretch of the centerline is implied at the macro-scale, except that 


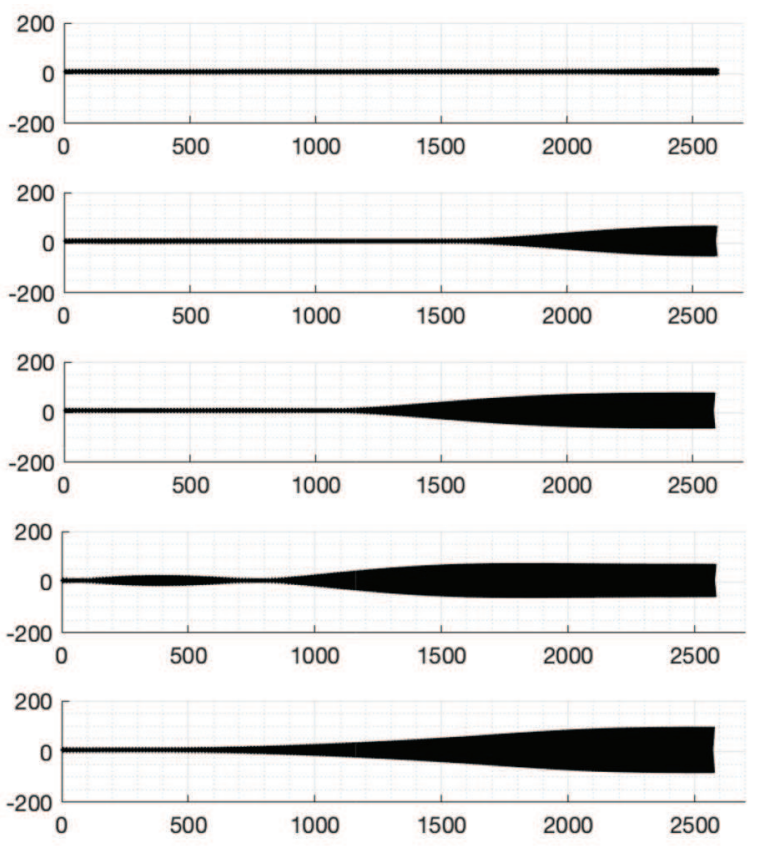

Figure 23. Hammer test for a pantographic beam with perfect pivots, 200 cells and triangle-shaped impulse. Stroboscopic plots of the current shape, for $2 r=1 \mathrm{~s}$, at time instants $t=0.2,0.7,1.2,1.7$, and $2.2 \mathrm{~s}(\Delta t=0.05 \mathrm{~s})$. Transversal displacements are multiplied by a factor of 500 to make plots more readable.

mentioned previously owing to shear-rotational springs. Hence, when perfect pivots are considered along with stiffnesses $a$ and $b$ in Table 1, the equivalent homogenized one-dimensional continuum exhibits an infinite internal length scale, which is roughly (there are, in fact, many of them) given by the square root of the ratio between the second gradient and first gradient stiffnesses (note that the stretch depends only on the first gradient of the placement field, whereas curvature and stretch gradient depend also on the second gradient of the placement field). This entails a high non-locality, namely that the deformation spreads uniformly in a wide area, which is our explanation of the absence of trails in the case of perfect pivots.

It is thus possible to conclude that trails observed for previous tests are directly related to the parameters $a$, $b$, and $c$ in Table 1 .

\section{Concluding remarks and future challenges}

Pantographic beams have the peculiarity of experiencing local large elongations associated to low deformation energies. As a consequence, one may expect the onset of peculiar propagating stretch profiles in dynamics which could possibly be persistent and produce traveling waves. The challenge of identifying a traveling wave in these structures presents non-trivial difficulties from the mathematical point of view. Indeed, in order to find traveling waves, one should find a wave form depending on one phase variable only and then solve a generalized eigenvalue problem to determine the possible propagation speeds together with the associated wave forms. This conceptual procedure applies more simply to equations having a field structure. Therefore, it may be useful to work out the homogenized equations associated with the discrete pantographic beam studied in the present work, which have been derived, albeit for a slightly simpler system, in [55]. This will be the subject of future investigations, as the asymptotic homogenization of the pantographic beam gives strongly nonlinear equations that generalize Euler's elastica.

The preliminary results on discrete pantographic beams that we have presented consist of some numerically calculated propagating profiles, that seem under specific conditions to propagate without a relevant shape change. We have identified a stretch profile in compression emerging during propagation, having a larger positive maximum value ahead and a smaller negative minimum behind with a tail, whose residual oscillations did not seem, for short traveled distances, to be associated with the unperturbed propagating profile. We have thus imposed on the free boundary an impulsive external load having these characteristics, i.e., zig-zag-shaped. 
Although we could not find evidence of persistent stretch waves, we are convinced that the ideas guiding our numerical experiments deserve to be applied more extensively because, as mentioned previously, the problem of identifying a persistent propagating stretch wave is not easy. It is for this reason that the obtained results also suggest that the homogenized continuum equations must be studied in order to determine with analytical or semi-analytical methods whether propagating wave profiles could exist and possibly exhibit a solitary-like pattern. Indeed, exploring the multitude of possible situations through numerical computations guided by a trial-and-error method is much more difficult than being guided by the understanding that one might get with a synthetic continuum model [64-67].

Aimed at guiding the mathematical determination of solitary-like waves, we examined the interference and superposition of two nonlinear profiles originating from left and right end points of the pantographic beam. The obtained results indicate that a more in-depth theoretical study is required: in fact, the two profiles travel, respectively, from the left to the right and vice versa without any apparent relevant interaction. We expect that this result may be confirmed by the study of the homogenized continuum equations derived in previous works.

The persistence of the propagating profile with no disturbances has been studied by varying the size of the pantographic beam and the duration of the externally applied impulse. Albeit the presented results are rather preliminary and a deeper analysis is required to reach a conclusive assessment of the nature of nonlinear waves in pantographic beams, we are laid to conjecture that zig-zag persistent propagating profiles could exist and depend critically on the propagating velocity and on the amplitudes of their maximum and minimum peaks. Should these conjectures be confirmed, one could deduce that the nonlinear dynamics of pantographic beams shares the extreme exoticity and peculiar mechanical behavior with the statics of pantographic beams. Owing to the results reported in Figure 14, one cannot obtain a definite answer to the raised question concerning the existence of traveling waves: in fact, an energy spillover from the original wave form to a modulated wave queue is observed. This fact could mean either that the conjectured profile is not of solitary type, or that we could not find the correct amplitude-speed relationship that assures its unperturbed propagation.

It is worth remarking that the analysis presented so far has considered three kinds of external loading. First, a triangular load in time was considered. Then, a trapezoidal load was considered. Finally, a load was considered such that it resembles, up to a velocity, the modified propagating wave shape obtained by using the triangular external load. The immediate next steps for future numerical investigations will consist of the analysis of wave propagation when amplitude-speed parameters are varied for the impulse profiles considered here. Such a parametric analysis can be made more efficient once longer pantographic beams and initial-boundary value problems with non-zero initial displacements and velocities will be incorporated within the numerical time integration scheme. In fact, one could apply iteratively the numerical initial-boundary problem solving scheme giving as an input for each iteration the evolved wave form calculated at the end of the previous iteration step. In other words, we may use the numerical solver as a filter that iteratively selects the wave form until a traveling wave is obtained.

Future outlooks also include the quantification of the dependence of the numerical dissipation on the parameters $\beta_{0}$ and $\beta_{1}$. Please note that, by changing $\beta_{0}$ and $\beta_{1}$, generally speaking, the accuracy and stability of the method may be affected negatively (this is very likely, actually, and Casciaro's scheme is indeed based on this) to an extent that should be quantified, especially for nonlinear systems. Thus, as in the solution approach we do not enforce any energy balance, this shall affect the conservation of energy. A systematic study on the effect of the parameters $\beta_{0}$ and $\beta_{1}$ is non-trivial and goes beyond the scopes of the present paper. We thus leave it for future studies.

We remark that it is possible to retrieve the forward Euler's quadrature method by fixing $\beta_{0}=1, \beta_{1}=0$, and considering $t=t_{1}$, which corresponds to $\xi=1$, in (10). Analogously, it is possible to retrieve the backward Euler's quadrature method by fixing $\beta_{0}=0, \beta_{1}=1$, and considering $t=t_{1}$, which corresponds to $\xi=1$, in (10). We also remark that, although Euler's methods are retrieved considering $\xi=1$ in (10), the interpolation in (10) is quadratic rather than constant, as it usually implied when dealing with Euler's methods. Looking at (11), it is also easy to conclude that Casciaro's method, because of the constraint $\beta_{0}+\beta_{1}=1$, falls within the class of generalized $\alpha$ methods. In addition, although non-optimal values for the parameters $\alpha_{0}$ and $\alpha_{1}$ may be chosen in (12) to match the standard Newmark- $\beta$ scheme, the main feature of this scheme, namely the firstorder approximation of velocities with respect to time, such that the acceleration appears, has no immediate connection with the scheme employed in this article.

We also note that trails similar to those observed for $c>0$, namely such that the first peak is followed by a tail, are visible also in the ingenious analytical solutions found by E. Schrödinger, in terms of Bessel functions, 
for the problem of the vibrations of a linear infinite chain of molecules. A translation into the English language of this article by E. Schrödinger, originally in German, has recently been made available, see [68].

\section{Funding}

The authors disclosed receipt of the following financial support for the research, authorship, and/or publication of this article: Emilio Turco was supported by the University of Sassari (Fondo di Ateneo per la ricerca 2020). Emilio Barchiesi was supported by the Instituto de Investigación Científica de la Universidad de Lima (Proyectos de Investigación 2021).

\section{Notes}

1. The reader who wishes to deepen the adopted modeling strategy will find many more details in [39].

2. Nodes are sketched in Figure 1 as small circles located at the intersection points between the beam-like elements.

3. Within this contribution, we assign the same extensional and bending stiffnesses to all the beam-like elements, i.e., uniform and balanced pantographic structures are considered, and we assign, for each unit cell and within each cell, the same pivots' torsional stiffness $c$. These hypotheses, obviously, can be easily removed to generalize the model.

4. We note that, while in principle both the stiffness matrix and the reaction could be assembled using a blind element-by-element procedure, the efficiency of the algorithm in terms of the occupied memory can be remarkably increased by taking into account the symmetry and the sparsity of the stiffness matrix to keep in memory its entries in a compact way.

5. We indeed remark that the size of the pivots may be varying along the system, in space. As an instance, given a base material, pivots, modeled in three dimensions as homogeneous cylinders, could have a varying radius. In statics, such a case has been studied in [69], where a (constrained) optimization of pivot diameters has been carried out. Note that pivot heights must be considered uniform under the plane motion assumption. Henceforth, the pivots will not only be considered as having the same stiffness, as mentioned earlier, but will also be considered as having the same mass. Physically, having in mind their realization into cylinders, this means that they are all made with the same material and present the same geometrical dimensions.

6. This is proved, e.g., by its low number of citations in the Google Scholar database: only 32 as of 28 December 2020).

7. The idea underlying this interpolation is analogous to that leading to the use of B-spline interpolation, which is at the basis of modern NURBS. It is worth remarking that, in 1985, this interpolation was used by Aristodemo [70] within the formulation of an efficient finite element for two-dimensional elastic problems.

8. Although not described in this work for the sake of brevity, Casciaro's stepwise strategy can be easily adapted for including dissipative forces, see, e.g., [71-73].

9. Please note that, without much more effort, more complex dependencies could be considered, such as $\mathbf{d}=\mathbf{d}(\mathbf{u}, \dot{\mathbf{u}})$. Also note that, indeed, such a case cannot be reconducted to the case $\mathbf{d}=\mathbf{d}(\dot{\mathbf{u}})$ (redefining the dissipation force and the structural reaction of course) when $\mathbf{d}$ cannot be additively decomposed into a term depending only on the displacement vector and a term depending only on the velocity vector.

\section{References}

[1] Alibert, JJ, Seppecher, P and dell'Isola, F. Truss modular beams with deformation energy depending on higher displacement gradients. Math Mech Solids 2003; 8(1): 51-73.

[2] dell'Isola, F, Seppecher, P, Spagnuolo, M, et al. Advances in pantographic structures: Design, manufacturing, models, experiments and image analyses. Continuum Mech Thermodyn 2019; 31(4): 1231-1282.

[3] dell'Isola, F, Seppecher, P, Alibert, JJ, et al. Pantographic metamaterials: An example of mathematically driven design and of its technological challenges. Continuum Mech Thermodyn 2019; 31(4): 851-884.

[4] Placidi, L, Barchiesi, E, Turco, E, et al. A review on 2D models for the description of pantographic fabrics. $Z$ angew Math Phys 2016; 67(121): 1-20.

[5] Barchiesi, E, and Placidi, L. A review on models for the 3D statics and 2D dynamics of pantographic fabrics. In Sumbatyan MA (ed.) Wave Dynamics and Composite Mechanics for Microstructured Materials and Metamaterials (Advanced Structured Materials, Vol. 59). Singapore: Springer, 2017, pp. 239-258.

[6] Barchiesi, E, Spagnuolo, M, and Placidi, L. Mechanical metamaterials: A state of the art. Math Mech Solids 2018; 24(1): 212-234

[7] dell'Isola, F, Giorgio, I, Pawlikowski, M, et al. Large deformations of planar extensible beams and pantographic lattices: Heuristic homogenisation, experimental and numerical examples of equilibrium. Proc R Soc Lond A Math Phys Eng Sci 2016; 472(2185): $1-23$. 
[8] Giorgio, I, Rizzi, NL, and Turco, E. Continuum modelling of pantographic sheets for out-of-plane bifurcation and vibrational analysis. Proc R Soc Lond A Math Phys Eng Sci 2017; 473(2207): 1-21.

[9] Giorgio, I, Della Corte, A, and dell'Isola, F. Dynamics of 1D nonlinear pantographic continua. Nonlin Dynam 2017; 88(1): 21-31.

[10] dell'Isola, F, Della Corte, A, Giorgio, I, et al. Pantographic 2D sheets: Discussion of some numerical investigations and potential applications. Int J Non-Lin Mech 2016; 80: 200-208.

[11] dell'Isola, F, Giorgio, I, and Andreaus, U. Elastic pantographic 2D lattices: A numerical analysis on static response and wave propagation. Proc Estonian Acad Sci 2015; 64(3): 219-225.

[12] Laudato, M, and Barchiesi, E. Non-linear dynamics of pantographic fabrics: Modelling and numerical study. In: Wave Dynamics, Mechanics and Physics of Microstructured Metamaterials. Cham: Springer, 2019, pp. 241-254.

[13] Abali, BE, and Barchiesi, E. Additive manufacturing introduced substructure and computational determination of metamaterials parameters by means of the asymptotic homogenization. Continuum Mech Thermodyn 2020; DOI: 10.1007/s00161-020-00941-w.

[14] Hild, F, Misra, A, and dell'Isola, F. Multiscale DIC applied to pantographic structures. Experimental mechanics. Exp Mech 2021; 61(2): 431-443.

[15] Boutin, C, dell'Isola, F, Giorgio, I, et al. Linear pantographic sheets. Asymptotic micro-macro models identification. Math Mech Complex Syst 2017; 5(2): 127-162.

[16] Laudato, M, Manzari, L, Scerrato, D, et al. Spectral properties of 2D pantographic metamaterial: Experimental results. Mech Res Commun 2020; 109: 103613

[17] Golaszewski, M, Grygoruk, R, Giorgio, I, et al. Metamaterials with relative displacements in their microstructure: Technological challenges in 3D printing, experiments and numerical predictions. Continuum Mech Thermodyn 2019; 31(4): 1015-1034.

[18] Misra, A, Lekszycki, T, Giorgio, I, et al. Pantographic metamaterials show atypical Poynting effect reversal. Mech Res Commun 2018; 89: 6-10.

[19] Nejadsadeghi, N, De Angelo, M, Drobnicki, M, et al. Parametric experimentation on pantographic unit cells reveals local extremum configuration. Exp Mech 2019; 59: 927-939.

[20] Tran, CA, Golaszewski, M, and Barchiesi, E. Symmetric-in-plane compression of polyamide pantographic fabrics: Modelling, experiments and numerical exploration. Symmetry 2020; 12(693): 1-17.

[21] Timofeev, D, Barchiesi, E, Misra, A, et al. Hemivariational continuum approach for granular solids with damage-induced anisotropy evolution. Math Mech Solids 2020; DOI: 10.1177/1081286520968149.

[22] Wang, D, Nejadsadeghi, N, Misra, A, et al. Local rotational dynamics reveals strain dependence in amorphous particle packings. Bull Amer Phys Soc 2021; in press.

[23] Nejadsadeghi, N, and Misra, A. Role of higher-order inertia in modulating elastic wave dispersion in materials with granular microstructure. Int J Mech Sci 2020; 185: 105867.

[24] Giorgio I, dell'Isola F, and Misra A. Chirality in $2 \mathrm{~d}$ cosserat media related to stretch-micro-rotation coupling with links to granular micromechanics. Int J Solids Struct 2020; 202: 28-38.

[25] Placidi, L, Andreaus, U, Della Corte, A, et al. Gedanken experiments for the determination of two-dimensional linear second gradient elasticity coefficients. Z angew Math Phys 2015; 66(6): 3699-3725.

[26] Placidi, L, Andreaus, U, and Giorgio, I. Identification of two-dimensional pantographic structure via a linear D4 orthotropic second gradient elastic model. J Eng Math 2017; 103(1): 1-21.

[27] Laudato, M, Manzari, L, Barchiesi, E, et al. First experimental observation of the dynamical behavior of a pantographic metamaterial. Mech Res Commun 2018; 94: 125-127.

[28] Turco, E. Stepwise analysis of pantographic beams subjected to impulsive loads. Math Mech Solids 2020; DOI: $10.1177 / 1081286520938841$.

[29] Turco E. A numerical survey of nonlinear dynamical responses of discrete pantographic beams. Continuum Mech Thermodyn 2021; doi: 10.1007/s00161-021-00989-2: 1-21.

[30] Baroudi, D, Giorgio, I, Battista, A et al. Nonlinear dynamics of uniformly loaded elastica: Experimental and numerical evidence of motion around curled stable equilibrium configurations. Z angew Math Mech 2019; 99(7): 1-20.

[31] Giorgio, I. A discrete formulation of Kirchhoff rods in large-motion dynamics. Math Mech Solids 2020; DOI: $10.1177 / 1081286519900902$.

[32] Eremeyev, V, and dell'Isola, F. A note on reduced strain gradient elasticity. In: Altenbach, H, Pouget, J, Rousseau, M, et al. (eds.) Generalized Models and Non-classical Approaches in Complex Materials 1. Cham: Springer, 2018, pp. 301-310.

[33] Eremeyev, VA, dell'Isola, F, Boutin, C, et al. Linear pantographic sheets: Existence and uniqueness of weak solutions. J Elasticity 2018; 132(2): 175-196.

[34] Turco, E, Giorgio, I, Misra, A, et al. King post truss as a motif for internal structure of (meta)material with controlled properties. R Soc Open Sci 2017; 4: 171153.

[35] Seppecher, P, Alibert, JJ, and dell'Isola, F. Linear elastic trusses leading to continua with exotic mechanical interactions. $J$ Phys Conf Ser 2011; 319(1): 012018.

[36] Giorgio, I, and Del Vescovo, D. Energy-based trajectory tracking and vibration control for multi-link highly flexible manipulators. Math Mech Complex Syst 2019; 7(2): 159-174.

[37] Giorgio, I, and Del Vescovo, D. Non-linear lumped-parameter modeling of planar multi-link manipulators with highly flexible arms. Robotics 2018; 7(60): 1-13. 
[38] Hencky, H. Über die angenäherte Lösung von Stabilitätsproblemen im Raum mittels der elastischen Gelenkkette. PhD Thesis, Engelmann, 1921.

[39] Turco, E, dell'Isola, F, Cazzani, A, et al. Hencky-type discrete model for pantographic structures: numerical comparison with second gradient continuum models. $Z$ angew Math Phys 2016; 67(85): 1-28.

[40] Turco, E, Golaszewski, M, Cazzani, A, et al. Large deformations induced in planar pantographic sheets by loads applied on fibers: Experimental validation of a discrete Lagrangian model. Mech Res Commun 2016; 76: 51-56.

[41] Capobianco, G, and Eugster, SR. Time finite element based Moreau-type integrators. Int J Numer Meth Eng 2018; 114 : 215 -231.

[42] Wriggers, P. Nonlinear Finite Element Methods. Berlin: Springer, 2008.

[43] Turco, E, Barcz, K, Pawlikowski, M, et al. Non-standard coupled extensional and bending bias tests for planar pantographic lattices. Part I: Numerical simulations. $Z$ angew Math Phys 2016; 67(122): 1-16.

[44] Turco, E, Barcz, K, and Rizzi, NL. Non-standard coupled extensional and bending bias tests for planar pantographic lattices. Part II: Comparison with experimental evidence. Z angew Math Phys 2016; 67(123): 1-16.

[45] Turco, E, and Rizzi, NL. Pantographic structures presenting statistically distributed defects: Numerical investigations of the effects on deformation fields. Mech Res Commun 2016; 77: 65-69.

[46] Turco, E, Golaszewski, M, Giorgio, I, et al. Pantographic lattices with non-orthogonal fibres: Experiments and their numerical simulations. Composites B Eng 2017; 118: 1-14.

[47] Turco, E, and Barchiesi, E. Equilibrium paths of Hencky pantographic beams in a three-point bending problem. Math Mech Complex Syst 2019; 7(4): 287-310.

[48] Turco, E. Numerically driven tuning of equilibrium paths for pantographic beams. Continuum Mech Thermodyn 2019; 31: 1941-1960.

[49] Eugster, SR, and Harsch, J. A variational formulation of classical nonlinear beam theories. In: Abali, BE and Giorgio, I (eds.) Developments and Novel Approaches in Nonlinear Solid Body Mechanics. Berlin: Springer, 2020, pp. 95-121.

[50] Harsch, J, and Eugster, SR. Finite element analysis of planar nonlinear classical beam theories. In: Abali BE and Giorgio I (eds.) Developments and Novel Approaches in Nonlinear Solid Body Mechanics. Berlin: Springer, 2020, pp. $123-157$.

[51] Capobianco, G, Eugster, SR, and Winandy, T. Modeling planar pantographic sheets using a nonlinear Euler-Bernoulli beam element based on B-spline functions. Proc Appl Math Mech 2018; 18(1): 1-2.

[52] Turco, E, Barchiesi, E, Giorgio, I, et al. A Lagrangian Hencky-type non-linear model suitable for metamaterials design of shearable and extensible slender deformable bodies alternative to Timoshenko theory. Int J Non-Lin Mech 2020; 123 : 103481.

[53] Turco, E, Misra, A, Pawlikowski, M, et al. Enhanced Piola-Hencky discrete models for pantographic sheets with pivots without deformation energy: numerics and experiments. Int J Solids Struct 2018; 147: 94-109.

[54] Barchiesi, E, dell'Isola, F, Laudato, M, et al. A 1D continuum model for beams with pantographic microstructure: Asymptotic micro-macro identification and numerical results. In: Advances in of Microstructured Media and Structures. Cham: Springer, 2018, pp. 43-74.

[55] Barchiesi, E, Eugster, SR, Placidi, L, et al. Pantographic beam: A complete second gradient 1D-continuum in plane. $Z$ angew Math Phys 2019; 70: 135.

[56] dell'Isola, F, Steigmann, D, Della Corte, A, et al. Discrete and Continuum Models for Complex Metamaterials. Cambridge: Cambridge University Press, 2020.

[57] Katsikadelis, JT. A new direct time integration method for the equations of motion in structural dynamics. $Z$ angew Math Mech 2013; 94(9): 757-774.

[58] de Miranda, S, Mancuso, M, and Ubertini, F. Time discontinuous Galerkin methods with energy decaying correction for nonlinear elastodynamics. Int J Numer Meth Eng 2010; 83: 323-347.

[59] Bathe, KJ, and Noh, G. Insight into an implicit time integration scheme for structural dynamics. Comput Struct 2012; 98-99: $1-6$.

[60] Casciaro, R. Time evolutional analysis of nonlinear structures. Meccanica 1975; 3(X): 156-167.

[61] Casciaro, R. An optimal time discretization method in structural analysis. Technical Report 161, Istituto di Scienza delle costruzioni, Università degli Studi di Roma, 1974.

[62] Riks, E. The application of Newton's method to the problem of elastic stability. J Appl Mech Trans ASME Ser E 1972; 39(4): 1060-1065.

[63] Barchiesi, E, Eugster, SR, dell'Isola, F, et al. Large in-plane elastic deformations of bi-pantographic fabrics: Asymptotic homogenization and experimental validation. Math Mech Solids 2020; 25(3): 739-767.

[64] Shirani, M, and Steigmann, DJ. A Cosserat model of elastic solids reinforced by a family of curved and twisted fibers. Symmetry 2020; 12(7): 1133.

[65] Steigmann, DJ. Theory of elastic solids reinforced with fibers resistant to extension, flexure and twist. Int J Non-Lin Mech 47; 7: 734-742.

[66] Steigmann, DJ, and dell'Isola, F. Mechanical response of fabric sheets to three-dimensional bending, twisting, and stretching. Acta Mechanica Sinica 2015; 31(3): 373-382.

[67] Steigmann, DJ. Two-dimensional models for the combined bending and stretching of plates and shells based on three-dimensional linear elasticity. Int J Eng Sci 46; 7: 654-676.

[68] Mühlich, U, Abali, BE, and dell'Isola, F. Commented translation of Erwin Schrödinger's paper "On the dynamics of elastically coupled point systems” (Zur Dynamik Elastisch Gekoppelter Punktsysteme). Math Mech Solids 2021; 26(1): $133-147$. 
[69] Desmorat, B, Spagnuolo, M, and Turco, E. Stiffness optimization in nonlinear pantographic structures. Math Mech Solids 2020; 25(11): 2252-2262.

[70] Aristodemo, M. A high-continuity finite element model for two-dimensional elastic problems. Comput Struct 1985; 21(5): 987-993.

[71] Andreaus, U, Baragatti, P, and Placidi, L. Experimental and numerical investigations of the responses of a cantilever beam possibly contacting a deformable and dissipative obstacle under harmonic excitation. Int J Non-Lin Mech 2016; 80: 96-106.

[72] Lekszycki, T, Olhoff, N, and Pedersen, JJ. Modelling and identification of viscoelastic properties of vibrating sandwich beams. Composite Struct 1992; 22(1): 15-31.

[73] dell'Isola, F, Rosa, L, and Wozniak, C. Dynamics of solids with microperiodic nonconnected fluid inclusions. Arch Appl Mech 1997; 67: 215-228 